\title{
Mössbauer Spectrometry
}

\author{
Brent Fultz \\ Department of Applied Physics and Materials Science \\ California Institute of Technology \\ Pasadena, California 91125
}

\begin{abstract}
Mössbauer spectrometry gives electronic, magnetic, and structural information from within materials. A Mössbauer spectrum is an intensity of $\gamma$-ray absorption versus energy for a specific resonant nucleus such as ${ }^{57} \mathrm{Fe}$ or ${ }^{119} \mathrm{Sn}$. For one nucleus to emit a $\gamma$-ray and a second nucleus to absorb it with efficiency, both nuclei must be embedded in solids, a phenomenon known as the "Mössbauer effect." Mössbauer spectrometry looks at materials from the "inside out," where "inside" refers to the resonant nucleus.

Mössbauer spectra give quantitative information on "hyperfine interactions," which are small energies from the interaction between the nucleus and its neighboring electrons. The three hyperfine interactions originate from the electron density at the nucleus (the isomer shift), the gradient of the electric field (the nuclear quadrupole splitting), and the unpaired electron density at the nucleus (the hyperfine magnetic field). Over the years, methods have been refined for using these three hyperfine interactions to determine valence and spin at the resonant atom. Even when the hyperfine interactions are not easily interpreted, they can often be used reliably as "fingerprints" to identify the different local chemical environments of the resonant atom, usually with a good estimate of their fractional abundances. Mössbauer spectrometry is useful for quantitative phase analyses or determinations of the concentrations of resonant element in different phases, even when the phases are nanostructured or amorphous.
\end{abstract}

Most Mössbauer spectra are acquired with simple laboratory equipment and a radioisotope source, but the recent development of synchrotron instrumentation now allow for measurements on small $10 \mu \mathrm{m}$ samples, which may be exposed to extreme environments of pressure and temperature. Other capabilities include measurements of the vibrational spectra of the resonant atoms, and coherent scattering and diffraction of nuclear radiation.

This article is not a review of the field, but an instructional reference that explains principles and practices, and gives the working materials scientist a basis for evaluating whether or not Mössbauer spectrometry may be useful for a research problem. A few representative materials studies are presented.

Please cite this work as follows:

Brent Fultz, "Mössbauer Spectrometry", in Characterization of Materials. Elton Kaufmann, Editor (John Wiley, New York, 2011). 


\section{Table of Contents}

Introduction

Principles of the Method

Nuclear Excitations

The Mössbauer Effect

Overview of Hyperfine Interactions

Recoil-Free Fraction

Isomer Shift

Electric Quadrupole Splitting

Hyperfine Magnetic Field Splitting

More Exotic Measurable Quantities

(Relaxation Phenomena, Phonons, Coherence and Diffraction)

Practical Aspects of the Method

Radioisotope Sources

Detectors for Radioisotope Source Experiments

Synchrotron Sources

Valence and Spin Determination

Phase Analysis

Solutes in bcc Fe Alloys

Crystal Defects and Nano-Particles

Data Analysis and Initial Interpretation

Sample Preparation

Literature Cited

Key References

Internet Resources 


\section{Mössbauer Spectrometry}

\section{INTRODUCTION}

Mössbauer spectrometry is based on the quantummechanical "Mössbauer effect," which provides a nonintuitive link between nuclear and solid-state physics. Mössbauer spectrometry measures the spectrum of energies at which specific nuclei absorb $\gamma$ rays. Curiously, for one nucleus to emit a $\gamma$ ray and a second nucleus to absorb it with efficiency, the atoms containing the two nuclei must be bonded chemically in solids. A young R. L. Mössbauer observed this efficient $\gamma$-ray emission and absorption process in ${ }^{191} \mathrm{Ir}$, and explained why the nuclei must be embedded in solids. Mössbauer spectrometry is now performed primarily with the nuclei ${ }^{57} \mathrm{Fe},{ }^{119} \mathrm{Sn},{ }^{151} \mathrm{Eu}$, ${ }^{121} \mathrm{Sb}$, and ${ }^{161} \mathrm{Dy}$. Mössbauer spectra can be obtained with other nuclei, but only if the experimenter can accept short radioisotope half-lives, cryogenic temperatures, and the preparation of radiation sources in hot cells.

Most applications of Mössbauer spectrometry in materials science utilize "hyperfine interactions," in which the electrons around a nucleus perturb the energies of nuclear states. Hyperfine interactions cause very small perturbations of $10^{-9}$ to $10^{-7} \mathrm{eV}$ in the energies of Mössbauer $\gamma$ rays. For comparison, the $\gamma$ rays themselves have energies of $10^{4}$ to $10^{5} \mathrm{eV}$. Surprisingly, these small hyperfine perturbations of $\gamma$-ray energies can be measured easily, and with high accuracy, using a low-cost Mössbauer spectrometer.

Interpretations of Mössbauer spectra have few parallels with other methods of materials characterization. Perhaps NMR spectrometry is the best analogy, although the excitation energies are very different. A Mössbauer spectrum looks at a material from the "inside out," where "inside" means the Mössbauer nucleus. The method is often useful because nuclear energy levels are altered by hyperfine interactions between the nucleus and its nearby electrons. With some interpretation, these hyperfine interactions can reveal the local atomic structure or electronic structure around the resonant Mössbauer atom. The important hyperfine interactions originate with the electron density at the nucleus, the gradient of the electric field at the nucleus, or the unpaired electron spins at the nucleus. These three hyperfine interactions are called the "isomer shift" (IS), "electric quadrupole splitting" (EQS), and "hyperfine magnetic field" (HMF), respectively.

The viewpoint from the nucleus is sometimes too small to address problems in the microstructure of materials. Over the past five decades, however, there has been considerable effort to learn how the three hyperfine interactions respond to the environment around the nucleus. In general, it is found that Mössbauer spectrometry is best for identifying the electronic or magnetic structure at the Mössbauer atom itself, such as its valence, spin state, or magnetic moment. The Mössbauer effect is sensitive to the arrangements of surrounding atoms, however, because the local crystal structure will affect the electronic or magnetic structure at the resonant nucleus. Different chemical and structural environments around the nucleus can often be assigned to specific hyperfine interactions. In such cases, measuring the fractions of nuclei with different hyperfine interactions is equivalent to measuring the fractions of the various chemical and structural environments in a material. Phase fractions and solute distributions, for example, can be determined in this way.

Other applications of the Mössbauer effect utilize its sensitivity to vibrations in solids, its timescale for scattering, or its coherence. To date these phenomena have seen little use outside the international community of a few hundred Mössbauer spectroscopists. Nevertheless, some new applications for them have recently become possible with the advent of synchrotron sources for Mössbauer spectrometry.

This unit is not a review of the Mössbauer spectrometry, but an instructional reference that gives the working materials scientist a basis for evaluating whether or not Mössbauer spectrometry may be useful for a research problem. There have been a number of books written about the Mössbauer effect and its spectroscopies (see Key References). Most include reviews of materials research. These reviews typically demonstrate applications of the measurable quantities in Mössbauer spectrometry, and provide copious references.

Recent research publications on Mössbauer spectrometry of materials have involved, in descending order in terms of numbers of papers: oxides, metals and alloys, organometallics, glasses, and minerals. For some problems, materials characterization by Mössbauer spectrometry is now "routine." A few representative applications to materials studies are presented. These applications were chosen in part according to the taste of the author, who makes no claim to have reviewed the literature of approximately 50,000 publications utilizing the Mössbauer effect (see Internet Resources for Mössbauer Effect Data Center Web site).

\section{PRINCIPLES OF THE METHOD} Nuclear Excitations

Many properties of atomic nuclei and nuclear matter are well established, but these properties are generally not well known by materials scientists. However, since Mössbauer spectrometry measures transitions between states of nuclei, some knowledge of nuclear properties is necessary to understand the measurements.

A nucleus can undergo transitions between quantum states, much like the electrons of an atom, and doing so involves large changes in energy. For example, the first excited state of ${ }^{57} \mathrm{Fe}$ is $14.41 \mathrm{keV}$ above its ground state. The Mössbauer effect is sometimes called "nuclear resonant $\gamma$-ray scattering" because it involves the emission of a $\gamma$ ray from an excited nucleus, followed by the absorption of this $\gamma$ ray by a second nucleus, which becomes excited. The scattering is called "resonant" because the phase and energy relationships for the $\gamma$-ray emission and absorption processes are much the same as for two coupled harmonic oscillators.

The state of a nucleus is described in part by the quantum numbers $E, I$, and $I_{z}$, where $E$ is energy and $I$ is the nuclear spin with orientation $I_{z}$ along a $z$ axis. In addition to these three internal nuclear coordinates, to understand the Mössbauer effect we also need spatial coordinates, $\mathrm{X}$, for the nuclear center of mass as the 
nucleus moves through space or vibrates in a crystal lattice. These center-of-mass coordinates are decoupled from the internal excitations of the nucleus.

The internal coordinates of the nucleus are mutually coupled. For example, the first excited state of the nucleus ${ }^{57} \mathrm{Fe}$ has spin $I=3 / 2$. For $I=3 / 2$, there are four possible values of $I_{z}$, namely, $-3 / 2,-1 / 2,+1 / 2$, and $+3 / 2$. The ground state of ${ }^{57} \mathrm{Fe}$ has $I=1 / 2$ and two allowed values of $I_{z}$. In the absence of hyperfine interactions to lift the energy degeneracies of spin levels, all allowed transitions between these spin levels will occur at the same energy, giving a total cross-section for nuclear absorption, $\sigma_{0}$, of $2.57 \times 10^{-18} \mathrm{~cm}^{2}$. Although $\sigma_{0}$ is smaller by a factor of 100 than a typical projected area of an atomic electron cloud, $\sigma_{0}$ is much larger than the characteristic size of the nucleus. It is also hundreds of times larger than the cross-section for scattering a $14.41-\mathrm{keV}$ photon by the atomic electrons at ${ }^{57} \mathrm{Fe}$.

The characteristic lifetime of the excited state of the ${ }^{57} \mathrm{Fe}$ nucleus, $\tau$, is $141 \mathrm{~ns}$, which is relatively long. An ensemble of independent ${ }^{57} \mathrm{Fe}$ nuclei that are excited simultaneously, by a flash of synchrotron light, for example, will decay at various times, $t$, with the probability per unit time of $1 / \tau \exp (-t / \tau)$. The time uncertainty of the nuclear excited state, $\tau$, is related to the energy uncertainty of the excited state, $\Delta E$, through the uncertainty relationship, $\hbar \sim \Delta E \tau$. For $\tau=141 \mathrm{~ns}$, the uncertainty relationship provides $\Delta E=4.7 \times 10^{-9} \mathrm{eV}$. This is remarkably small - the energy of the nuclear excited state is extremely precise in energy. A nuclear resonant $\gamma$ ray emission or absorption has an oscillator quality factor, $Q$, of $3 \times 10^{12}$. The purity of phase of the $\gamma$ ray is equally impressive. In terms of information technology, it is possible in principle to transmit high-quality audio recordings of all the Beethoven symphonies on a single Mössbauer $\gamma$-ray photon. The technology for modulating and demodulating this information remains problematic, however.

For a single type of nuclear transition, the energy dependence of the cross-section for Mössbauer scattering is of Lorentzian form, with a width determined by the small lifetime broadening of the excited state energy

$$
\sigma_{j}(E)=\frac{\sigma_{0} p_{j}}{1+\left(\frac{E-E_{j}}{\Gamma / 2}\right)^{2}}
$$

where for ${ }^{57} \mathrm{Fe}, \Gamma=\Delta E=4.7 \times 10^{-9} \mathrm{eV}$, and $E_{j}$ is the mean energy of the nuclear level transition $(14.41 \mathrm{keV})$. Here $p_{\mathrm{j}}$ is the fraction of nuclear absorptions that will occur with energy $E_{j}$. In the usual case where the energy levels of the different Mössbauer nuclei are inequivalent and the nuclei scatter independently, the total cross section is

$$
\sigma(E)=\sum_{j} \sigma_{j}(E)
$$

A Mössbauer spectrometry measurement is usually designed to measure the energy dependence of the total cross-section, $\sigma(E)$, which is often a sum of Lorentzian functions of natural line width $\Gamma$.

It is sometimes possible to measure coherent Mössbauer scattering. Here the total intensity, $I(E)$, from a sample is not the sum of independent intensity contributions from individual nuclei. One considers instead the total wave, $\Psi(\mathbf{r}, E)$, at a detector located at $\mathbf{r}$. The total wave, $\Psi(\mathbf{r}, E)$, is the sum of the scattered waves from individual nuclei, $j$

$$
\Psi(\vec{r}, E)=\sum_{j} \psi_{j}(\vec{r}, E)
$$

Equation 3 is fundamentally different from Equation 2, since wave amplitudes rather than intensities are added. Since we add the individual $\Psi_{j}$, it is necessary to account precisely for the phases of the waves scattered by the different nuclei. Interpretations of coherent scattering data tend to involve some advanced physics (Hannon and Trammell, 1969; van Bürck, et al., 1978; Sturhahn and Gerdau, 1994).

\section{The Mössbauer Effect}

Up to this point, we have assumed it possible for a second nucleus to become excited by absorbing the energy of a $\gamma$ ray emitted by a first nucleus. This nuclear resonance was observed before Mössbauer's discovery, but the experiments suffered from a well recognized difficulty. As mentioned above, the energy precision of a nuclear excited state can be on the order of $10^{-8} \mathrm{eV}$. This is an extremely small energy target to hit with an incident $\gamma$ ray. At room temperature, for example, vibrations of the nuclear center of mass have energies of $2.5 \times 10^{-2} \mathrm{eV} /$ atom. If changes in the vibrational energy of the nucleus occurred during $\gamma$-ray emission, the $\gamma$ ray would be far too imprecise in energy to be absorbed by the sharp resonance of a second nucleus. In classical mechanics we expect such a change, since the emission of a $\gamma$ ray of momentum $p_{\gamma}=E_{\gamma} / c$ requires the recoil of the emitting system with an opposite momentum (where $E_{\gamma}$ is the $\gamma$-ray energy and $c$ is the speed of light). A mass, $m$, will recoil after such a momentum transfer, and the kinetic energy in the recoil, $E_{\text {recoil }}$, will detract from the $\gamma$-ray energy

$$
E_{\text {recoil }}=\frac{p_{\gamma}^{2}}{2 m}=\frac{E_{\gamma}^{2}}{2 m c^{2}}
$$

For the recoil of a single nucleus, we use the mass of a ${ }^{57} \mathrm{Fe}$ nucleus for $m$ in Equation 4, and find that $E_{\text {recoil }}=1.86 \times$ $10^{-3} \mathrm{eV}$. This is again many orders of magnitude larger than the energy precision required for the $\gamma$ ray to be absorbed by a second nucleus.

Rudolf Mössbauer's doctoral thesis project was to measure nuclear resonant scattering in ${ }^{191}$ Ir. His approach was to use thermal Doppler broadening of the emission line to compensate for the recoil energy. A few resonant nuclear absorptions could be expected this way. To his surprise, the number of resonant absorptions was large, and was even larger when his radiation source and absorber were cooled to liquid nitrogen temperature (where the thermal Doppler broadening is smaller). Adapting a theory developed by W. E. Lamb for neutron resonant scattering (Lamb, 1939), Mössbauer interpreted his observed effect and obtained the equivalent of Equation 19, below. Mössbauer further realized that by using small mechanical motions, he could provide Doppler shifts to the $\gamma$-ray energies and tune through the nuclear resonance. He did so, and observed a 
spectrum without thermal Doppler broadening. In 1961, R. L. Mössbauer won the Nobel prize for physics. He was 32 .

Mössbauer discovered (Mössbauer, 1958) that under appropriate conditions, the mass, $m$, in Equation 4 could be equal to the mass of the entire crystal, not just one nucleus. In such a case, the recoil energy is trivially small, the energy of the outgoing $\gamma$ ray is precise to better than $10^{-9} \mathrm{eV}$, and the $\gamma$ ray can be absorbed by exciting a second nucleus. The question is now how the mass, $m$, could be so large. The idea is that the nuclear mass is attached rigidly to the mass of the crystal. This sounds rather unrealistic, of course, and a better model is that the ${ }^{57} \mathrm{Fe}$ nucleus is attached to the crystal mass by a spring. This is the problem of a simple harmonic oscillator, or equivalently the Einstein model of a solid with Einstein frequency $\omega_{\mathrm{E}}$. The oscillator is quantized, however, and sometimes the $\gamma$-ray emission occurs with a change in the quantum state, but sometimes the state is unchanged.

Eventually, the momentum of the $\gamma$-ray emission, $p_{\gamma}=$ $E_{\gamma} / c$, will be taken up by the recoil of the crystal as a whole. However, it is possible that the energy levels of a simple harmonic oscillator (comprising the Mössbauer nucleus bound to the other atoms of the crystal lattice) could be changed by the $\gamma$-ray emission. An excitation of this oscillator would depreciate the $\gamma$-ray energy by $n \hbar \omega_{\mathrm{E}}$ if $n$ phonons are excited during the $\gamma$-ray emission. Since $\hbar \omega_{\mathrm{E}}$ is on the order of $10^{-2} \mathrm{eV}$, any change in oscillator energy would spoil the possibility for a subsequent resonant absorption. In essence, quantized changes in the oscillator excitation (or phonons in a periodic solid) replace the classical recoil energy (Equation 4) that spoils the energy precision of the emitted $\gamma$ ray. The key to the Mössbauer effect, however, is the probability that phonon excitation does not occur during $\gamma$-ray emission.

Before $\gamma$-ray emission, the wavefunction of the nuclear center of mass is $\psi_{\mathrm{i}}(\mathrm{X})$, which can also be represented in momentum space through the Fourier transformation

$$
\phi_{i}(\vec{p})=\frac{1}{\sqrt{2 \pi \hbar}} \int_{-\infty}^{+\infty} \mathrm{e}^{-\frac{\mathrm{i} \vec{p} \cdot \vec{X}^{\prime}}{\hbar}} \psi_{i}\left(\vec{X}^{\prime}\right) \mathrm{d} \vec{X}^{\prime}
$$

or

$$
\psi_{i}(\vec{X})=\frac{1}{\sqrt{2 \pi \hbar}} \int_{-\infty}^{+\infty} \mathrm{e}^{+\frac{\mathrm{i} \vec{p} \cdot \vec{X}}{\hbar}} \phi_{i}(\vec{p}) \mathrm{d} \vec{p}
$$

The momentum space representation can handily accommodate the impulse of the $\gamma$-ray emission, giving the final state of the nuclear center of mass, $\psi_{\mathrm{f}}(X)$. Recall that the impulse is the time integral of the force, $F=\mathrm{d} p / \mathrm{d} t$, which equals the change in momentum. (The analog to impulse in momentum space is a translation in real-space, such as $X \rightarrow X-X_{0}$.) This corresponds to obtaining a final state by a shift in origin of an initial eigenstate. With the emission of a $\gamma$ ray having momentum $p_{\gamma}$, we obtain the final state wave function from the initial eigenstate through a shift of origin in momentum space, $\phi_{\mathrm{i}}(p) \rightarrow \phi_{\mathrm{i}}\left(p-p_{\gamma}\right)$. We interpret the final state in real-space, $\psi_{\mathrm{f}}(\mathrm{X})$, with Equation 6

$$
\psi_{f}(\vec{X})=\frac{1}{\sqrt{2 \pi \hbar}} \int_{-\infty}^{+\infty} \mathrm{e}^{+\frac{\mathrm{i}(\vec{p}+\vec{p} \gamma) \cdot \vec{X}}{\hbar}} \phi_{i}(\vec{p}) \mathrm{d} \vec{p}
$$

Now, substituting Equation 5 into Equation 7

$$
\psi_{f}(\vec{X})=\frac{1}{2 \pi \hbar} \int_{-\infty}^{+\infty} \mathrm{e}^{+\frac{\mathrm{i}(\vec{p}+\vec{p} \gamma) \cdot \vec{X}}{\hbar}} \int_{-\infty}^{+\infty} \mathrm{e}^{-\frac{\mathrm{i} \vec{p} \cdot \vec{X}^{\prime}}{\hbar}} \psi_{i}\left(\vec{X}^{\prime}\right) \mathrm{d} \vec{X}^{\prime} \mathrm{d} \vec{p}
$$

Isolating the integration over momentum, $p$

$$
\psi_{f}(\vec{X})=\frac{1}{2 \pi \hbar} \int_{-\infty}^{+\infty} \mathrm{e}^{+\frac{\mathrm{i} \vec{p} \gamma \cdot \vec{X}}{\hbar}} \psi_{i}\left(\vec{X}^{\prime}\right)\left[\int_{-\infty}^{+\infty} \mathrm{e}^{+\frac{\mathrm{i} \vec{p} \cdot\left(\vec{X}-\vec{X}^{\prime}\right)}{\hbar}} \mathrm{d} \vec{p}\right] \mathrm{d} \vec{X}^{\prime}
$$

The integration over $p$ gives a Dirac delta function (times $2 \pi \hbar)$

$$
\begin{gathered}
\psi_{f}(\vec{X})=\int_{-\infty}^{+\infty} \mathrm{e}^{+\frac{\mathrm{i} \vec{\gamma} \gamma \cdot \vec{X}}{\hbar}} \psi_{i}\left(\vec{X}^{\prime}\right) \delta\left(\vec{X}-\vec{X}^{\prime}\right) \mathrm{d} \vec{X}^{\prime} \\
\psi_{f}(\vec{X})=\mathrm{e}^{+\frac{\mathrm{i} \vec{p} \gamma \cdot \vec{X}}{\hbar}} \psi_{i}(\vec{X})
\end{gathered}
$$

The exponential in Equation 11 is a translation of the eigenstate, $\Psi_{\mathrm{i}}(X)$, in position for a fixed momentum transfer, $-p_{\gamma}$. It is similar to the translation in time, $t$, of an eigenstate with fixed energy, $E$, which is $\exp (-\mathrm{i} E t / \hbar)$ or a translation in momentum for a fixed spatial translation, $X_{0}$, which is $\exp \left(-\mathrm{i} p X_{0} / \hbar\right)$. (If the initial state is not an eigenstate, $p_{\gamma}$ in Equation 11 must be replaced by an operator.)

For the nuclear center-of-mass wavefunction after $\gamma$-ray emission, we seek the amplitude of the initial state wavefunction that remains in the final state wavefunction. In Dirac notation

$$
\langle i \mid f\rangle \equiv \int_{-\infty}^{+\infty} \psi_{i}^{*}(\vec{X}) \psi_{f}(\vec{X}) \mathrm{d} \vec{X}
$$

Substituting Equation 11 into Equation 12, and using Dirac notation

$$
\langle i \mid f\rangle \equiv\left\langle i\left|\mathrm{e}^{+\frac{\mathrm{i} \vec{p} \gamma \cdot \vec{x}}{\hbar}}\right| i\right\rangle
$$

Using the convention for the $\gamma$-ray wavevector, $k_{y} \equiv 2 \pi v / c$ $=E_{\gamma} / \hbar c$

$$
\langle i \mid f\rangle \equiv\left\langle i\left|\mathrm{e}^{+\mathrm{i} \vec{k}_{\gamma} \cdot \vec{X}}\right| i\right\rangle
$$

The inner product $\langle i \mid f\rangle$ is the projection of the initial state of the nuclear center of mass on the final state after emission of the $\gamma$ ray. It provides the probability that there is no change in the state of the nuclear center of mass caused by $\gamma$-ray emission. The probability of this "recoilless emission," $f$, is the square of the matrix element of Equation 14, normalized by all possible changes of the center-of-mass eigenfunctions

$$
\begin{array}{r}
f=\frac{\left|\left\langle i\left|\mathrm{e}^{+\mathrm{i} \vec{k}_{\gamma} \cdot \vec{X}}\right| i\right\rangle\right|^{2}}{\sum_{j}\left|\left\langle j\left|\mathrm{e}^{+\mathrm{i} \vec{k}_{\gamma} \cdot \vec{X}}\right| i\right\rangle\right|^{2}} \\
f=\frac{\left|\left\langle i\left|\mathrm{e}^{+\mathrm{i} \vec{k}_{\gamma} \cdot \vec{X}_{1}}\right| i\right\rangle\right|^{2}}{\sum_{j}\left\langle i\left|\mathrm{e}^{-\mathrm{i} \vec{k}_{\gamma} \cdot \vec{X}}\right| j\right\rangle\left\langle j\left|\mathrm{e}^{+\mathrm{i} \vec{k}_{\gamma} \cdot \vec{X}}\right| i\right\rangle}
\end{array}
$$


Using the closure relation $\Sigma_{\mathrm{j}}|j><j|=1$, and the normalization $<i \mid i>=1$, Equation 16 becomes

$$
f=\left|\left\langle i\left|\mathrm{e}^{+\mathrm{i} \vec{k}_{\gamma} \cdot \vec{X}}\right| i\right\rangle\right|^{2}
$$

The quantity $f$ is the "recoil-free-fraction." It is the probability that, after the $\gamma$ ray removes momentum $p_{\gamma}$ from the nuclear center of mass, there will be no change in the lattice state function involving the nuclear center of mass. In other words, $f$ is the probability that a $\gamma$ ray will be emitted with no energy loss to phonons. A similar factor is required for the absorption of a $\gamma$ ray by a nucleus in a second crystal (e.g., the sample). The evaluation of $f$ is straightforward for the ground state of the Einstein solid. The ground state wavefunction is

$$
\psi_{\mathrm{CM}}^{0}(X)=\left(\frac{m \omega_{\mathrm{E}}}{\pi \hbar}\right)^{\frac{1}{4}} \mathrm{e}^{-\frac{m \omega_{\mathrm{E}} X^{2}}{2 \hbar}}
$$

Inserting Equation 18 into Equation 17, and evaluating the integral (which is the Fourier transform of a Gaussian function)

$$
f=\mathrm{e}^{-\frac{\hbar^{2} k_{\gamma}^{2}}{2 m \hbar \omega_{\mathrm{E}}}}=\mathrm{e}^{-\frac{E_{\mathrm{R}}}{\hbar \omega_{\mathrm{E}}}}=\mathrm{e}^{-k_{\gamma}^{2}\langle X\rangle^{2}}
$$

where $E_{\mathrm{R}}$ is the recoil energy of a free ${ }^{57} \mathrm{Fe}$ nucleus, and $\left\langle X^{2}\right\rangle$ is the mean-squared displacement of the nucleus when bound in an oscillator. It is somewhat more complicated to use a Debye model for calculating $f$ with a distribution of phonon energies (Mössbauer, 1958). When the lattice dynamics are known, computer calculations can be used to obtain $f$ from the full phonon spectrum of the solid, including the phonon polarizations. These more detailed calculations essentially confirm the result of Equation 19. The only nontrivial point is that low-energy phonons do not alter the result significantly. The recoil of a single nucleus does not couple effectively to long wavelength phonons, and there are few of them, so their excitation is not a problem for recoilless emission.

The condition for obtaining a significant number of "recoilless" $\gamma$-ray emissions is that the characteristic recoil energy of a free nucleus, $E_{\mathrm{R}}$, is smaller than, or on the order of, the energy of the short wavelength phonons in the solid. These phonon energies are typically estimated from the Debye or Einstein temperatures of the solid to be a few tens of meV. Since $E_{\mathrm{R}}=1.86 \times 10^{-3} \mathrm{eV}$ for ${ }^{57} \mathrm{Fe}$, this condition is satisfied nicely. It is not uncommon for most of the $\gamma$-ray emissions or absorptions from ${ }^{57} \mathrm{Fe}$ to be recoilfree. It is helpful that the energy of the $\gamma$ ray, $14.41 \mathrm{keV}$, is relatively low. Higher-energy $\gamma$ rays cause $E_{\mathrm{R}}$ to be large, as seen by the quadratic relation in Equation 4. Energies of most $\gamma$ rays are far greater than $14 \mathrm{keV}$, so Mössbauer spectrometry is not practical for most nuclear transitions.

\section{Overview of Hyperfine Interactions}

Given the existence of the Mössbauer effect, the question remains as to what it can do. The answer is given in two parts: what are the phenomena that can be measured, and then what do these measurables tell us about materials? The four standard measurable quantities are the recoil-free fraction $(f)$ and the three hyperfine interactions: the isomer shift, the electric quadrupole splitting, and the hyperfine magnetic field. To date, the three hyperfine interactions have proved the most useful measurable quantities for the characterization of materials by Mössbauer spectrometry. This overview provides a few rules of thumb as to the types of information that can be obtained from hyperfine interactions. The section below (see More Exotic Measurable Quantities) describes quantities that are measurable, but which have seen fewer applications so far. For specific applications of hyperfine interactions for studies of materials, see Practical Aspects of the Method.

The isomer shift is the easiest hyperfine interaction to understand. It is a direct measure of electron density, albeit at the nucleus and away from the electron density responsible for chemical bonding between the Mössbauer atom and its neighbors. The isomer shift changes with the valence of the Mössbauer atom such as ${ }^{57} \mathrm{Fe}$ or ${ }^{119} \mathrm{Sn}$. It is possible to use the isomer shift to estimate the fraction of Mössbauer isotope in different valence states, which may originate from different crystallographic site occupancies or from the presence of multiple phases in a sample. Valence analysis is often straightforward, and is probably the most common type of service work that Mössbauer spectroscopists provide for other materials scientists. The isomer shift has proven most useful for studies of ionic or covalently bonded materials such as oxides and minerals. Unfortunately, although the isomer shift is in principle sensitive to local atomic coordinations, it has usually not proven useful for structural characterization of materials, except when changes in valence are involved. The isomer shifts caused by most local structural distortions are generally too small to be useful.

Electric field gradients (EFG) are often correlated to isomer shifts. The existence of an $\mathrm{EFG}$ requires an asymmetric (i.e., noncubic) electronic environment around the nucleus, however, and this usually correlates with the local atomic structure. Again, like the isomer shift, the EFG has proven most useful for studies of oxides and minerals. Although interpretations of the EFG are not so straightforward as the isomer shift, the EFG is more capable of providing information about the local atomic coordination of the Mössbauer isotope. For ${ }^{57} \mathrm{Fe}$, the shifts in peak positions caused by the EFG tend to be comparable to, or larger than, those caused by the isomer shift.

While isomer shifts are universal, hyperfine magnetic fields (HMF) are confined to ferro-, ferri-, or antiferromagnetic materials. However, while isomer shifts tend to be small, HMFs usually provide large and distinct shifts of Mössbauer peaks. Because their effects are so large and varied, HMFs often permit detailed materials characterizations by Mössbauer spectrometry. For bodycentered cubic (bcc) Fe alloys, it is known how most solutes in the periodic table alter the magnetic moments and HMFs at neighboring Fe atoms, so it is often possible to measure the distribution of HMFs and determine distributions of solute atoms about ${ }^{57} \mathrm{Fe}$ atoms. In magnetically ordered $\mathrm{Fe}$ oxides, the distinct HMFs allow for ready identification of phase, sometimes more readily than by x-ray diffractometry.

Even in cases where fundamental interpretations of Mössbauer spectra are impossible, the identification of the local chemistry around the Mössbauer isotope is often 
possible by "fingerprint" comparisons with known standards. Mössbauer spectrometers tend to have similar instrument characteristics, so quantitative comparisons with published spectra are often possible. A literature search for related Mössbauer publications is usually enough to locate standard spectra for comparison. The Mössbauer Effect Data Center (see Internet Resources) is another resource that can provide this information.

\section{Recoil-Free Fraction}

An obvious quantity to measure with the Mössbauer effect is its intensity, given by Equation 19 as the recoil-free fraction, $f$. The recoil-free fraction is reminiscent of the Debye-Waller factor for X-ray diffraction. It is large when the lattice is stiff and $\omega_{\mathrm{E}}$ is large. Like the Debye-Waller factor, $f$ is a weighted average over all phonons in the solid. Unlike the Debye-Waller factor, however, $f$ must be determined from measurements with only one value of wavevector $k$, which is of course $k_{\gamma}$.

It is difficult to obtain $f$ from a single absolute measurement, since details about the sample thickness and absorption characteristics must be known accurately. Comparative studies may be possible with in situ experiments where a material undergoes a phase transition from one state to another while the macroscopic shape of the specimen is unchanged.

The usual way to determine $f$ for a single-phase material is by measuring Mössbauer spectral areas as a function of temperature, $T$. Equation 19 shows that the intensity of the Mössbauer effect will decrease with $\left\langle X^{2}\right\rangle$, the meansquared displacement of the nuclear motion. The $\left\langle X^{2}\right\rangle$ increases with $T$, so measurements of spectral intensity versus $T$ can provide the means for determining $f$, and hence the Debye or Einstein temperature of the solid.

Another effect that occurs with temperature provides a measure of $\left\langle v^{2}\right\rangle$, where $v$ is the velocity of the nuclear center of mass. This effect is sometimes called the "second order Doppler shift," but it originates with special relativity. When a nucleus emits a $\gamma$ ray and loses energy, its mass is reduced slightly. The phonon occupation numbers do not change, but the phonon energy is increased slightly owing to the diminished mass. This reduces the energy available to the $\gamma$-ray photon. This effect is usually of greater concern for absorption by the specimen, for which the energy shift is

$$
E_{\text {therm }}=-\frac{1}{2} \frac{\left\langle v^{2}\right\rangle}{c^{2}} E_{0}
$$

The thermal shift scales with the thermal kinetic energy in the sample, which is essentially a measure of temperature. For ${ }^{57} \mathrm{Fe}, E_{\text {therm }}=-7.3 \times 10^{-4} \mathrm{~mm} / \mathrm{s} \mathrm{K}$.

\section{Isomer Shift}

The peaks in a Mössbauer spectrum undergo observable shifts in energy when the Mössbauer atom is in different materials. These shifts originate from a hyperfine interaction involving the nucleus and the inner electrons of the atom. These "isomer shifts" are in proportion to the electron density at the nucleus. Two possibly unfamiliar concepts underlie the origin of the isomer shift. First, some atomic electron wavefunctions are actually present inside the nucleus. Second, the nuclear radius is different in the nuclear ground and excited states.

In solving the Schrödinger equation for radial wavefunctions of electrons around a point nucleus, it is found that for $r \rightarrow 0$ (i.e., toward the nucleus) the electron wavefunctions go as $r^{l}$, where $l$ is the angular momentum quantum number of the electron. For $s$ electrons $(1 s, 2 s, 3 s$, $4 s$, etc.) with $l=0$, the electron wavefunction is quite large at $r=0$. It might be guessed that the wavefunctions of $s$ electrons could make some sort of sharp wiggle so they go to zero inside the nucleus, but this would cost too much kinetic energy. The $s$ electrons (and some relativistic $p$ electrons) are actually present inside the nucleus. Furthermore, the electron density is essentially constant across the small size of the nucleus.

The overlap of the $s$-electron wavefunction with the finite nucleus provides a Coulomb perturbation that lowers the nuclear energy levels. If the excited state and groundstate energy levels were lowered equally, however, the energy of the nuclear transition would be unaffected, and the emitted (or absorbed) $\gamma$ ray would have the same energy. It is well known that the radius of an atom changes when an electron enters an excited state. The same type of effect occurs for nuclei-the nuclear radius is different for the nuclear ground and excited states. For ${ }^{57} \mathrm{Fe}$, the effective radius of the nuclear excited state, $R_{\mathrm{ex}}$, is smaller than the radius of the ground state, $R_{\mathrm{g}}$ but for ${ }^{119} \mathrm{Sn}$ it is the other way around. For the overlap of a finite nucleus with a constant charge density, the total electrostatic attraction is stronger when the nucleus is smaller. This leads to a difference in energy between the nuclear excited state and ground state in the presence of a constant electron density $|\psi(0)|^{2}$. This shift in transition energy will usually be different for nuclei in the radiation source and nuclei in the sample, giving the following shift in position of the absorption peak in the measured spectrum

$$
\Delta E_{\mathrm{IS}}=C Z e^{2}\left(R_{\mathrm{ex}}^{2}-R_{\mathrm{g}}^{2}\right)\left|\psi_{\text {sample }}(0)-\psi_{\text {source }}(0)\right|^{2}
$$

The factor $C$ depends on the shape of the nuclear charge distribution, which need not be uniform or spherical. The sign of Equation 21 for ${ }^{57} \mathrm{Fe}$ is such that with an increasing s-electron density at the nucleus, the Mössbauer peaks will be shifted to more negative velocity. For ${ }^{119} \mathrm{Sn}$, the difference in nuclear radii has the opposite sign. With increasing $s$-electron density at a ${ }^{119} \mathrm{Sn}$ nucleus, the Mössbauer peaks shift to more positive velocity.

There remains another issue in interpreting isomer shifts, however. In the case of $\mathrm{Fe}$, the $3 d$ electrons are expected to partly screen the nuclear charge from the $4 \mathrm{~s}$ electrons. An increase in the number of $3 d$ electrons at an ${ }^{57} \mathrm{Fe}$ atom will therefore increase this screening, reducing the $s$-electron density at the ${ }^{57} \mathrm{Fe}$ nucleus and causing a more positive isomer shift. The $s$-electron density at the nucleus is therefore not simply proportional to the number of valence $s$ electrons at the ion. The effect of this $3 d$ electron screening is large for ionic compounds (Gütlich, 1975). In these compounds there is a series of trend lines for how the isomer shift depends on the $4 s$ electron density, where the different trends correspond to the different number of $3 d$ electrons at the ${ }^{57} \mathrm{Fe}$ atom (Walker et al., 1961). With more $3 d$ electrons, the isomer shift is more 
positive, but also the isomer shift becomes less sensitive to the number of $4 s$ electrons at the atom. Determining the valence state of $\mathrm{Fe}$ atoms from isomer shifts is generally a realistic type of experiment, however (see Practical Aspects of the Method).

For metals it has been more recently learned that the isomer shifts do not depend on the $3 d$ electron density (Akai et al., 1986). In Fe alloys, the isomer shift corresponds nicely to the $4 \mathrm{~s}$ charge transfer, in spite of changes in the $3 d$ electrons at the $\mathrm{Fe}$ atoms. For the first factor in Equation 21, a proposed choice for ${ }^{57} \mathrm{Fe}$ is $\left[C Z e^{2}\left(R_{\mathrm{ex}}^{2}-R_{\mathrm{g}}^{2}\right)\right]=-0.24 \mathrm{a}_{0}^{3} \mathrm{~mm} / \mathrm{s} \quad$ (Akai et al., 1986), where $\mathrm{a}_{0}$ is the Bohr radius of $0.529 \AA$.

\section{Electric Quadrupole Splitting}

The isomer shift, described in the previous section, is an electric monopole interaction. There is no static dipole moment of the nucleus. The nucleus does have an electric quadrupole moment that originates with its asymmetrical shape. The asymmetry of the nucleus depends on its spin, which differs for the ground and excited states of the nucleus. In a uniform electric field, the shape of the nuclear charge distribution has no effect on the Coulomb energy. In an electric field gradient (EFG), however, there will be different interaction energies for different alignments of the electric quadrupole moment of the nucleus. An EFG generally involves a variation with position of the $x, y$, and $z$ components of the electric field vector. In specifying an $\mathrm{EFG}$, it is necessary to know, for example, how the $x$ component of the electric field, $V_{x}=\partial V / \partial x$ varies along the $y$ direction, $V_{y x} \equiv \partial^{2} V / \partial y \partial x[V(x, y, z)$ is the electric potential]. The EFG involves all such partial derivatives, and is a tensor quantity. In the absence of competing hyperfine interactions, it is possible to choose freely a set of principal axes so that the off-diagonal elements of the EFG tensor are zero. By convention, we label the principal axes such that $\left|V_{z z}\right|>\left|V_{y y}\right|>\left|V_{x x}\right|$. Furthermore, because the Laplacian of the potential vanishes, $V_{\mathrm{xx}}+V_{\mathrm{yy}}+V_{\mathrm{zz}}=0$, there are only two parameters required to specify the EFG. These are chosen to be $V_{\mathrm{zz}}$ and an asymmetry parameter, $\eta \equiv\left(V_{\mathrm{xx}}-V_{\mathrm{yy}}\right) / V_{\mathrm{zz}}$.

The isotopes ${ }^{57} \mathrm{Fe}$ and ${ }^{119} \mathrm{Sn}$ have an excited-state spin of $I=3 / 2$ and a ground-state spin of $1 / 2$. The shape of the excited nucleus is that of a prolate spheroid. This prolate spheroid will be oriented with its long axis pointing along the $z$ axis of the EFG when $I_{z}= \pm 3 / 2$. There is no effect from the sign of $I_{z}$, since inverting a prolate spheroid does not change its charge distribution. The $I_{z}= \pm 3 / 2$ states have a low energy compared to the $I_{\mathrm{z}}=1 / 2$ orientation of the excited state. In the presence of an EFG, the excited-state energy is split into two levels. Since $I_{z}= \pm 1 / 2$ for the ground state, however, the ground state energy is not split by the EFG. With an electric quadrupole moment for the excited state defined as $Q$, for ${ }^{57} \mathrm{Fe}$ and ${ }^{119} \mathrm{Sn}$ the quadrupole splitting of energy levels is

$$
\Delta E_{\mathrm{q}}=\frac{ \pm 1}{4} e Q V_{z z}\left(1+\frac{\eta^{2}}{3}\right)^{\frac{1}{2}}
$$

where often there is the additional definition $e q \equiv V_{\mathrm{zz}}$. The energy level diagram is shown in Figure 1. By definition, $\eta$ $<1$, so the asymmetry factor can vary only from 1 to 1.155 .
For ${ }^{57} \mathrm{Fe}$ and ${ }^{119} \mathrm{Sn}$, for which Equation 22 is valid, the asymmetry can usually be neglected, and the electric quadrupole interaction can be assumed to be a measure of $V_{\mathrm{zz}}$. Unfortunately, it is not possible to determine the sign of $V_{\mathrm{zz}}$ easily (although this has been done by applying high magnetic fields to the sample).

The EFG is zero when the electronic environment of the Mössbauer isotope has cubic symmetry. When the electronic symmetry is reduced, a single line in the Mössbauer spectrum appears as two lines separated in energy as described by Equation 22 (as shown in Fig. 1). When the ${ }^{57} \mathrm{Fe}$ atom has a $3 d$ electronic structure with orbital angular momentum, $V_{\mathrm{zz}}$ is large. High- and lowspin Fe complexes can be identified by differences in their electric quadrupole splitting. The electric quadrupole splitting is also sensitive to the local atomic arrangements, such as ligand charge and coordination, but this sensitivity is not possible to interpret by simple calculations. The ligand field gives an enhanced effect on the EFG at the nucleus because the electronic structure at the Mössbauer atom is itself distorted by the ligand. This effect is termed "Sternheimer antishielding," and enhances the EFG from the ligands by a factor of about 7 for ${ }^{57} \mathrm{Fe}$ (Watson and Freeman, 1967).

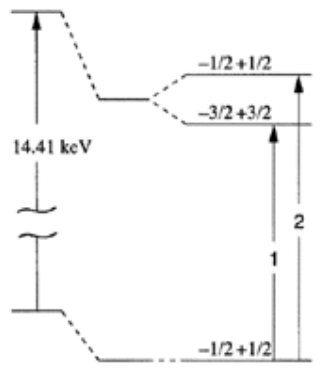

$\begin{array}{cc}\text { Isolated } & \text { Isomer } \\ \text { Nucleus } & \text { Shift }\end{array}$
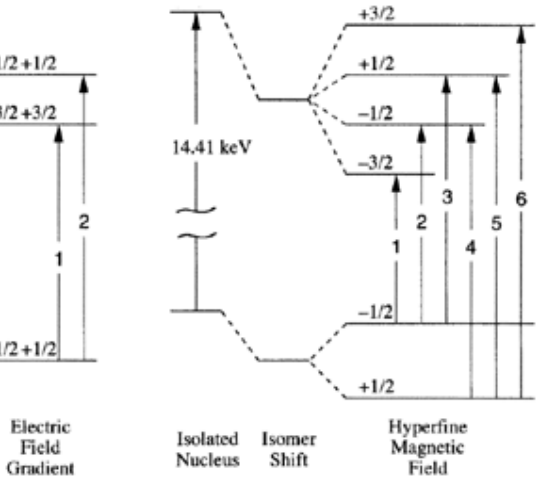

Figure 1. Energy level diagrams for ${ }^{57} \mathrm{Fe}$ in an electric field gradient (EFG; left) or hyperfine magnetic field (HMF; right). For an HMF at the sample, the numbers 1 to 6 indicate progressively more energetic transitions, which give experimental peaks at progressively more positive velocities. Sign convention is that an applied magnetic field along the direction of lattice magnetization will reduce the HMF and the magnetic splitting. The case where the nucleus is exposed simultaneously to an EFG and HMF of approximately the same energies is much more complicated than can be presented on a simple energy level diagram.

\section{Hyperfine Magnetic Field Splitting}

The nuclear states have spin, and associated magnetic dipole moments. The spins can be oriented with different projections along a magnetic field. The energies of nuclear transitions are therefore modified when the nucleus is in a magnetic field. The energy perturbations caused by this HMF are sometimes called the "nuclear Zeeman effect," in analogy with the more familiar splitting of energy levels of atomic electrons when there is a magnetic field at the atom.

A hyperfine magnetic field lifts all degeneracies of the spin states of the nucleus, resulting in separate transitions 
identifiable in a Mössbauer spectrum (see, e.g., Fig. 2). The $I_{\mathrm{z}}$ range from $-I$ to $+I$ in increments of 1 , being $\{-3 / 2,-1 / 2$, $+1 / 2,+3 / 2\}$ for the excited state of ${ }^{57} \mathrm{Fe}$ and $\{-1 / 2,+1 / 2\}$ for the ground state. The allowed transitions between ground and excited states are set by selection rules. For the M1 magnetic dipole radiation for ${ }^{57} \mathrm{Fe}$, six transitions are allowed: $\{(-1 / 2 \rightarrow-3 / 2)(-1 / 2 \rightarrow-1 / 2)(-1 / 2 \rightarrow+1 / 2)(+1 / 2 \rightarrow-1 / 2)$ $(+1 / 2 \rightarrow+1 / 2) \quad(+1 / 2 \rightarrow+3 / 2)\}$. The allowed transitions are shown in Figure 1. Notice the inversion in energy levels of the nuclear ground state.

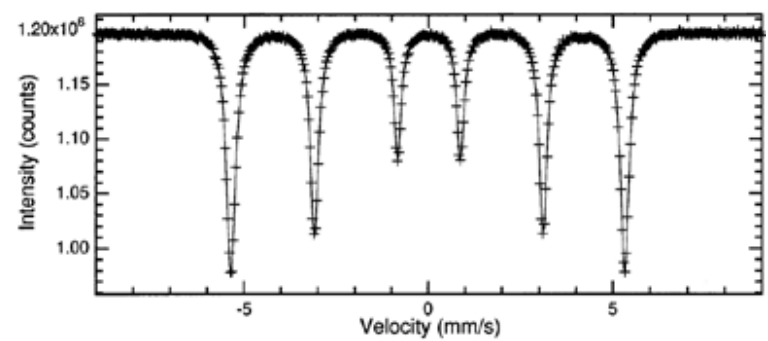

Figure 2. Mössbauer spectrum from bcc Fe. Data were acquired at $300 \mathrm{~K}$ in transmission geometry with a constant acceleration spectrometer (Ranger MS900). The points are the experimental data. The solid line is a fit to the data for six independent Lorentzian functions with unconstrained centers, widths, and depths. Also in the fit was a parabolic background function, which accounts for the fact that the radiation source was somewhat closer to the specimen at zero velocity than at the large positive or negative velocities. $A{ }^{57} \mathrm{Co}$ source in $\mathrm{Rh}$ was used, but the zero of the velocity scale is the centroid of the Fe spectrum itself. Separation between peaks 1 and 6 is $10.62 \mathrm{~mm} / \mathrm{s}$.

In ferromagnetic iron metal, the magnetic field at the ${ }^{57} \mathrm{Fe}$ nucleus, the HMF, is $33.0 \mathrm{~T}$ at $300 \mathrm{~K}$. The enormity of this HMF suggests immediately that it does not originate from the traditional mechanisms of solid-state magnetism. Furthermore, when an external magnetic field is applied to a sample of Fe metal, there is a decrease in magnetic splitting of the measured Mössbauer peaks. This latter observation shows that the HMF at the ${ }^{57} \mathrm{Fe}$ nucleus has a sign opposite to that of the lattice magnetization of $\mathrm{Fe}$ metal, so the HMF is given as $-33.0 \mathrm{~T}$.

It is easiest to understand the classical contributions to the HMF, denoted $H_{\text {mag }}, H_{\text {dip }}$ and $H_{\text {orb. }}$. The contribution $H_{\text {mag }}$ is the magnetic field from the lattice magnetization, $M$, which is $4 \pi M / 3$. To this contribution we add any magnetic fields applied by the experimenter, and we subtract the demagnetization caused by the return flux. Typically, $H_{\text {mag }}<+0.7 \mathrm{~T}$. The contribution $H_{\text {dip }}$ is the classical dipole magnetic field caused by magnetic moments at atoms near the Mössbauer nucleus. In Fe metal, $H_{\text {dip }}$ vanishes owing to cubic symmetry, but contributions of $+0.1 \mathrm{~T}$ are possible when neighboring $\mathrm{Fe}$ atoms are replaced with nonmagnetic solutes. Finally, $H_{\text {orb }}$ originates with any residual orbital magnetic moment from the Mössbauer atom that is not quenched when the atom is a crystal lattice. This contribution is about $+2 \mathrm{~T}$ (Akai, 1986), and it may not change significantly when Fe metal is alloyed with solute atoms, for example. These classical mechanisms make only minor contributions to the HMF.
The big contribution to the HMF at a Mössbauer nucleus originates with the "Fermi contact interaction." Using the Dirac equation, Fermi and Segre discovered a new term in the Hamiltonian for the interaction of a nucleus and an atomic electron

$$
\hbar_{\mathrm{FC}}=-\frac{8 \pi}{3} g_{\mathrm{e}} g_{\mathrm{N}} \mu_{\mathrm{e}} \mu_{\mathrm{N}} \mathbf{I} \cdot \mathbf{S} \delta(\mathbf{r})
$$

Here $\mathbf{I}$ and $\mathbf{S}$ are spin operators that act on the nuclear and electron wavefunctions, respectively, $\mu_{\mathrm{e}}$ and $\mu_{\mathrm{N}}$ are the electron and nuclear magnetons, and $\delta(\mathbf{r})$ ensures that the electron wavefunction is sampled at the nucleus. Much like the electron gyromagnetic ratio, $g_{e}$, the nuclear gyromagnetic ratio, $g_{\mathrm{N}}$, is a proportionality between the nuclear spin and the nuclear magnetic moment. Unlike the case for an electron, the nuclear ground and excited states do not have the same value of $g_{\mathrm{N}}$; that of the ground state of ${ }^{57} \mathrm{Fe}$ is larger by a factor of -1.7145 . The nuclear magnetic moment is $g_{\mathrm{N}} \mu_{\mathrm{N}} I$, so we can express the Fermi contact energy by considering this nuclear magnetic moment in an effective magnetic field, $H_{\text {eff }}$, defined as

$$
H_{\text {eff }}=\frac{8 \pi}{3} g_{\mathrm{e}} \mu_{\mathrm{e}} S|\psi(0)|^{2}
$$

where the electron spin is $\pm 1 / 2$, and $|\psi(0)|^{2}$ is the electron density at the nucleus. If two electrons of opposite spin have the same density at the nucleus, their contributions will cancel and $H_{\text {eff }}$ will be zero. A large HMF requires an unpaired electron density at the nucleus, expressed as $|S|>$ 0 .

The Fermi contact interaction explains why the HMF is negative in ${ }^{57} \mathrm{Fe}$. As described above (see Isomer Shift), only $s$ electrons of $\mathrm{Fe}$ have a substantial presence at the nucleus. The largest contribution to the ${ }^{57} \mathrm{Fe} \mathrm{HMF}$ is from $2 s$ electrons, however, which are spin-paired core electrons. The reason that spin-paired core electrons can make a large contribution to the HMF is that the $2 s \uparrow$ and $2 s \downarrow$ wavefunctions have slightly different shapes when the $\mathrm{Fe}$ atom is magnetic. The magnetic moment of $\mathrm{Fe}$ atoms originates primarily with unpaired $3 d$ electrons, so the imbalance in numbers of $3 d \uparrow$ and $3 d \downarrow$ electrons must affect the shapes of the paired $2 s \uparrow$ and $2 s \downarrow$, electrons.

These shapes of the $2 s \uparrow$ and $2 s \downarrow$ electron wavefunctions are altered by exchange interactions with the $3 d \uparrow$ and $3 d \downarrow$ electrons. The exchange interaction originates with the Pauli exclusion principle, which requires that a multielectron wavefunction be antisymmetric under the exchange of electron coordinates. The process of antisymmetrization of a multielectron wavefunction produces an energy contribution from the Coulomb interaction between electrons called the "exchange energy," which is the expectation value of the Coulomb energy for all pairs of electrons of like spin exchanged between their wavefunctions.

The net effect of the exchange interaction is to decrease the repulsive energy between electrons of like spin. In particular, the exchange interaction reduces the Coulomb repulsion between the $2 s \uparrow$ and $3 d \uparrow$ electrons, allowing the more centralized $2 s \uparrow$ electrons to expand outward away from the nucleus. The same effect occurs for the $2 s \downarrow$ and $3 d \downarrow$ electrons, but to a lesser extent because there are fewer $3 d \downarrow$ electrons than $3 d \uparrow$ electrons in ferromagnetic Fe. The result is a higher density of $2 s \downarrow$ than $2 s \uparrow$ electrons 
at the ${ }^{57} \mathrm{Fe}$ nucleus. The same effect occurs for the $1 s$ shell, and the net result is that the HMF at the ${ }^{57} \mathrm{Fe}$ nucleus is opposite in sign to the lattice magnetization (which is dominated by the $3 d \uparrow$ electrons). The $3 s$ electrons contribute to the HMF, but are at about the same mean radius as the $3 d$ electrons, so their spin unbalance at the ${ }^{57} \mathrm{Fe}$ nucleus is smaller. The $4 s$ electrons, on the other hand, lie outside the $3 d$ shell, and exchange interactions bring a higher density of $4 s \uparrow$ electrons into the ${ }^{57} \mathrm{Fe}$ nucleus, although not enough to overcome the effects of the $1 s \downarrow$ and $2 s \downarrow$ electrons. These $4 s$ spin polarizations are sensitive to the magnetic moments at nearest neighbor atoms, however, and provide a mechanism for the ${ }^{57} \mathrm{Fe}$ atom to sense the presence of neighboring solute atoms. This is described below (see Solutes in bcc Fe Alloys).

\section{More Exotic Measurable Quantities}

Relaxation Phenomena. Hyperfine interactions have natural time windows for sampling electric or magnetic fields. This time window is the characteristic time, $\tau_{\mathrm{hf}}$, associated with the energy of a hyperfine splitting, $\tau_{\mathrm{hf}}=$ $\hbar / E_{\mathrm{hf}}$. When a hyperfine electric or magnetic field undergoes fluctuations on the order of $\tau_{\mathrm{hf}}$ or faster, observable distortions appear in the measured Mössbauer spectrum. The lifetime of the nuclear excited state does not play a direct role in setting the timescale for observing such relaxation phenomena. However, the lifetime of the nuclear excited state does provide a reasonable estimate of the longest characteristic time for fluctuations that can be measured by Mössbauer spectrometry.

Sensitivity to changes in valence of the Mössbauer atom between $\mathrm{Fe}(\mathrm{II})$ and $\mathrm{Fe}$ (III) has been used in studies of the Verwey transition in $\mathrm{Fe}_{3} \mathrm{O}_{4}$, which occurs at $\sim 120 \mathrm{~K}$. Above the Verwey transition temperature the Mössbauer spectrum comprises two sextets, but when $\mathrm{Fe}_{3} \mathrm{O}_{4}$ is cooled below the Verwey transition temperature the spectrum becomes complex (Degrave et al., 1993).

Atomic diffusion is another phenomenon that can be studied by Mössbauer spectrometry (Ruebenbauer et al., 1994). As an atom jumps to a new site on a crystal lattice, the coherence of its $\gamma$-ray emission is disturbed. The shortening of the time for coherent $\gamma$-ray emission causes a broadening of the linewidths in the Mössbauer spectrum. In single crystals this broadening can be shown to occur by different amounts along different crystallographic directions, and has been used to identify the atom jump directions and mechanisms of diffusion in Fe alloys (Feldwisch et al., 1994; Vogl et al., 1994; Sepiol et al., 1996).

Perhaps the most familiar example of a relaxation effect in Mössbauer spectrometry is the superparamagnetic behavior of small particles. This phenomenon is described below (see Crystal Defects and Small Particles, Figure 9). A different example showing thermally-activated charge dynamics is presented in Figure 5.

Phonons. The phonon partial density of states (DOS) has recently become measurable by Mössbauer spectrometry. Technically, nuclear resonant scattering that occurs with the creation or annihilation of a phonon is inelastic scattering, and is therefore not the Mössbauer effect. However, techniques for measuring the phonon partial DOS have been developed as a capability of synchrotron radiation sources for Mössbauer scattering. The experiments are performed by detuning the incident photon energies above and below the nuclear resonance by $100 \mathrm{meV}$ or so. This range of energy is far beyond the energy width of the Mössbauer resonance or any of its hyperfine interactions. However, it is in the range of typical phonon energies. The inelastic spectra so obtained are called "partial" phonon densities of states because they involve the motions of only the Mössbauer nucleus. The experiments (Seto et al., 1995; Sturhahn et al., 1995; Fultz et al., 1997) are performed with incoherent scattering (a Mössbauer $\gamma$ ray into the sample, a conversion $x$ ray out), and are interpreted in the same way as incoherent inelastic neutron scattering spectra (Squires, 1978). Compared to this latter, more established technique, the inelastic nuclear resonant scattering experiments have the capability of working with much smaller samples, owing to the large cross-section for nuclear resonant scattering. The vibrational spectra of monolayers of ${ }^{57} \mathrm{Fe}$ atoms at interfaces of thin films and in nanoparticles have been measured, and shown to be quite different from spectra of bulk materials (Cuenya, 2007; Cuenya 2008).

Coherence and Diffraction. Mössbauer scattering can be coherent, meaning that the phase of the incident wave is in a precise relationship to the phase of the scattered wave. For coherent scattering, wave amplitudes are added (Equation 3) instead of independent photon intensities (Equation 2). For the isotope ${ }^{57} \mathrm{Fe}$, coherency occurs only in experiments where a $14.41 \mathrm{keV} \gamma$ ray is absorbed and a $14.41 \mathrm{keV} \gamma$ ray is reemitted through the reverse nuclear transition. The waves scattered by different coherent processes interfere with each other, either constructively or destructively. The interference between Mössbauer scattering and $\mathrm{x}$-ray Rayleigh scattering undergoes a change from constructive in-phase interference above the Mössbauer resonance to destructive out-of-phase interference below. This gives rise to an asymmetry in the peaks measured in an energy spectrum, first observed by measuring a Mössbauer energy spectrum in scattering geometry (Black and Moon, 1960).

Diffraction is a specialized type of interference phenomenon. Of particular interest to the physics of Mössbauer diffraction is a suppression of internal conversion processes when diffraction is strong. With multiple transfers of energy between forward and diffracted beams, there is a nonintuitive enhancement in the rate of decay of the nuclear excited state (Hannon and Trammell, 1969; van Bürck et al., 1978; Shvyd'ko and Smirnov, 1989), and a broadening of the characteristic linewidth. A fortunate consequence for highly perfect crystals is that with strong Bragg diffraction, a much larger fraction of the reemissions from ${ }^{57} \mathrm{Fe}$ nuclei occur by coherent $14.41 \mathrm{keV}$ emission. The intensities of Mössbauer diffraction peaks therefore become stronger and easier to observe. For solving unknown structures in materials or condensed matter, however, it is difficult to interpret the intensities of diffraction peaks when there are multiple scatterings. Quantification of diffraction intensities with kinematical theory is an advantage of performing Mössbauer diffraction experiments on polycrystalline samples. Such samples also avoid the broadening of features in the Mössbauer energy 
spectrum that accompanies the speedup of the nuclear decay. Unfortunately, without the dynamical enhancement of coherent decay channels, kinematical diffraction experiments on small crystals suffer a serious penalty in diffraction intensity. Powder diffraction patterns have not been obtained until recently (Stephens et al., 1994), owing to the low intensities of the diffraction peaks. Mössbauer diffraction from polycrystalline alloys does offer a new capability, however, of combining the spectroscopic capabilities of hyperfine interactions to extract a diffraction pattern from a particular chemical environment of the Mössbauer isotope (Stephens and Fultz, 1997; Lin and Fultz, 2003,2004).

\section{PRACTICAL ASPECTS OF THE METHOD}

\section{Radioisotope Sources}

The vast majority of Mössbauer spectra have been measured with instrumentation as shown in Figure 3. The spectrum is obtained by counting the number of $\gamma$-ray photons that pass through a thin specimen as a function of the $\gamma$-ray energy. At energies where the Mössbauer effect is strong, a dip is observed in the $\gamma$-ray transmission. The $\gamma$ ray energy is tuned with a drive that imparts a Doppler shift, $\Delta E$, to the $\gamma$ ray in the reference frame of the sample:

$$
\Delta E=\frac{v}{c} E_{\gamma}
$$

where $v$ is the velocity of the drive. A velocity of $10 \mathrm{~mm} / \mathrm{s}$ provides an energy shift, $\Delta E$, of $4.8 \times 10^{-7} \mathrm{eV}$ to a 14.41 $\mathrm{keV} \gamma$ ray of ${ }^{57} \mathrm{Fe}$. Recall that the energy width of the Mössbauer resonance is $4.7 \times 10^{-9} \mathrm{eV}$, which corresponds to $0.097 \mathrm{~mm} / \mathrm{s}$. An energy range of $10 \mathrm{~mm} / \mathrm{s}$ is usually more than sufficient to tune through the full Mössbauer energy spectrum of ${ }^{57} \mathrm{Fe}$ or ${ }^{119} \mathrm{Sn}$. It is conventional to present the energy axis of a Mössbauer spectrum in units of $\mathrm{mm} / \mathrm{s}$.

The equipment required for Mössbauer spectrometry is simple, and adequate instrumentation is often found in instructional laboratories for undergraduate physics students. In a typical coursework laboratory exercise, students learn the operation of the detector electronics and the spectrometer drive system in a few hours, and complete a measurement or two in about a week. (The understanding of the measured spectrum typically takes much longer.) Most components for the Mössbauer spectrometer in Figure 3 are standard items for x-ray detection and data acquisition. The items specialized for Mössbauer spectrometry are the electromagnetic drive and the radiation source. Abandoned electromagnetic drives and controllers are often found in university and industrial laboratories, and hardware manufactured since about 1970 by WissEl GmbH, Austin Science Associates, Ranger Scientific, Elscint, Ltd., and Renon are all capable of providing excellent results. Half-lives for radiation sources are: ${ }^{57} \mathrm{Co}, 271$ days, ${ }^{119 \mathrm{~m}} \mathrm{Sn}, 245$ days, ${ }^{151} \mathrm{Sm}, 93$ years, and ${ }^{125} \mathrm{Te}, 2.7$ years. A new laboratory setup for ${ }^{57} \mathrm{Fe}$ or ${ }^{119} \mathrm{Sn}$ work may require the purchase of a radiation source. Suppliers include Ritverc GmbH, Cyclotron Co., See Co. and Gamma-Lab Development S.L. Specifications for the purchase of a new Mössbauer source, besides activity level (typically 20 to $50 \mathrm{mCi}$ for ${ }^{57} \mathrm{Co}$ ), should include linewidth and sometimes levels of impurity radioisotopes.

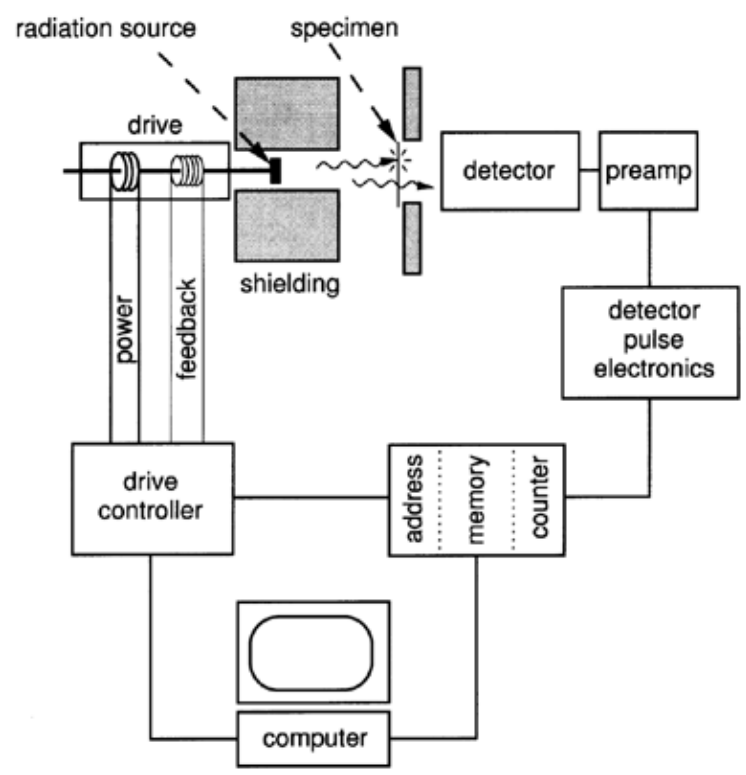

Figure 3. Transmission Mössbauer spectrometer. The radiation source sends $\gamma$ rays to the right through a thin specimen into a detector. The electromagnetic drive is operated with feedback control by comparing a measured velocity signal with a desired reference waveform. The drive is cycled repetitively, usually so the velocity of the source varies linearly with time (constant acceleration mode). Counts from the detector are accumulated repetitively in short time intervals associated with memory addresses of a multichannel scaler. Each time interval corresponds to a particular velocity of the radiation source. Typical numbers are 1024 data points of $50-\mu$ s time duration and a period of $20 \mathrm{~Hz}$.

Radiation sources for ${ }^{57} \mathrm{Fe}$ Mössbauer spectrometry use the ${ }^{57} \mathrm{Co}$ radioisotope. The unstable ${ }^{57} \mathrm{Co}$ nucleus absorbs an inner-shell electron, transmuting to ${ }^{57} \mathrm{Fe}$ and emitting a $122-\mathrm{keV} \gamma$ ray. The ${ }^{57} \mathrm{Fe}$ nucleus thus formed is in its first excited state, and decays about $141 \mathrm{~ns}$ later by the emission of a $14.41-\mathrm{keV} \gamma$ ray. This second $\gamma$ ray is the useful photon for Mössbauer spectrometry. While the $122 \mathrm{keV} \gamma$ ray can be used as a clock to mark the formation of the ${ }^{57} \mathrm{Fe}$ excited state, it is generally considered a nuisance in Mössbauer spectrometry, along with emissions from other contamination radioisotopes in the radiation source. A Mössbauer radiation source is prepared by diffusing the ${ }^{57} \mathrm{Co}$ isotope into a matrix material such as $\mathrm{Rh}$, so that atoms of ${ }^{57} \mathrm{Co}$ reside as dilute substitutional solutes on the fcc Rh crystal lattice. Being dilute, the ${ }^{57} \mathrm{Co}$ atoms have a neighborhood of pure $\mathrm{Rh}$, and therefore all ${ }^{57} \mathrm{Co}$ atoms have the same local environment and the same nuclear energy levels. They will therefore emit $\gamma$ rays of the same energy. Although radiation precautions are required for handling the source, the samples (absorbers) are not radioactive either before or after measurement in the spectrometer. 
The measured energy spectrum from the sample is convoluted with the energy spectrum of the radiation source. For a spectrum with sharp Lorentzian lines of natural linewidth, $\Gamma$ (see Equation 1), the convolution of the source and sample Lorentzian functions provides a measured Lorentzian function of full width at halfmaximum of $0.198 \mathrm{~mm} / \mathrm{s}$. An excellent ${ }^{57} \mathrm{Fe}$ spectrum from pure Fe metal over an energy range of $10 \mathrm{~mm} / \mathrm{s}$ may have linewidths of $0.23 \mathrm{~mm} / \mathrm{s}$, although instrumental linewidths of somewhat less than $0.3 \mathrm{~mm} / \mathrm{s}$ are not uncommon owing to technical problems with the purity of the radiation source and vibrations of the specimen or source.

\section{Detectors for Radioisotope Source Experiments}

The resonant $\gamma$ rays in Mössbauer spectrometry have relatively low energies, so conventional $\mathrm{x}$-ray detectors are used in many Mössbauer spectrometers. For ${ }^{57} \mathrm{Fe}$ spectroscopy, it is often convenient if the detector is transparent to the $122 \mathrm{keV}$ precursor $\gamma$ ray, hence reducing the nonresonant count rate. Scintillators should therefore be very thin. Gas-filled proportional counters are often convenient, and offer better energy resolution. Solid-state detectors are excellent for service in transmission Mössbauer spectrometers when the count rate is not excessive.

Although a highly monochromatic $\gamma$ ray from a first nucleus is required to excite a second Mössbauer nucleus, the subsequent decay of the second nucleus in the sample need not occur by the reemission of a $\gamma$ ray. In fact, for ${ }^{57} \mathrm{Fe}$ only $10.9 \%$ of the decays occur in this way. Most of the decays occur by "internal conversion" processes, where the energy of the nuclear excited state is transferred to the atomic electrons. These electrons typically leave the atom, or rearrange their atomic states to emit an $\mathrm{x}$ ray. These conversion electrons or conversion $\mathrm{x}$ rays can themselves be used for measuring a Mössbauer spectrum. The conversion electrons offer the capability for surface analysis of a material. If the electron detector has good energy resolution, the surface sensitivity of conversion electron Mössbauer spectrometry can be as small as a monolayer (Faldum et al., 1994; Stahl and Kankeleit, 1997; Kruijer et al., 1997).

A backscatter conversion electron detector that counts electrons emitted from the sample surface after resonant absorption is especially useful when thin samples are difficult to prepare. The most common backscatter conversion electron detector is a gas-filled proportional counter, with the sample itself sealing the flowing gas mixture of $\mathrm{He}+10 \% \mathrm{CH}_{4}$. These detectors tend to be flat, and for good signal-to-noise they should be a thin, perhaps 3-4 $\mathrm{mm}$, along the direction of the incident $\gamma$ ray. Even a thin layer of $\mathrm{He}$ gas at atmospheric pressure has good stopping power for conversion electrons, but by making the detector very thin, more the incident $\gamma$ ray beam will pass through the gas on its path to the sample surface. Typically, electrons of a wide range of energies are detected, providing a depth sensitivity for conversion electron Mössbauer spectrometry of $\sim 100 \mathrm{~nm}$ (Gancedo et al., 1991; Williamson, 1993). Conversion electron detection is often useful as a probe of the near-surface region of a sample.
Enrichment of the Mössbauer isotope is sometimes needed when the $2.2 \%$ natural abundance of ${ }^{57} \mathrm{Fe}$ is insufficient to provide a strong spectrum. Although ${ }^{57} \mathrm{Fe}$ is not radioactive, material enriched to $95 \%{ }^{57} \mathrm{Fe}$ costs approximately $\$ 5$ to $\$ 10$ per $\mathrm{mg}$, so specimen preparation usually involves only small quantities of isotope. Biochemical experiments often require growing organisms in the presence of ${ }^{57} \mathrm{Fe}$. This is common practice for studies on heme proteins, for example. For inorganic materials, it is sometimes possible to study dilute concentrations of $\mathrm{Fe}$ by isotopic enrichment. It is also common practice to use ${ }^{57} \mathrm{Fe}$ as an impurity, even when $\mathrm{Fe}$ is not part of the structure. Sometimes it is clear that the ${ }^{57} \mathrm{Fe}$ atom will substitute on the site of another transition metal, for example, and the local chemistry of this site can be studied with ${ }^{57} \mathrm{Fe}$ dopants.

The same type of doping experiments can be used with the ${ }^{57} \mathrm{Co}$ radioisotope, but this is not a common practice because it involves the preparation of radioactive materials. With ${ }^{57} \mathrm{Co}$ doping, the sample material itself serves as the radiation source, and the sample is moved with respect to a single-line absorber to acquire the Mössbauer spectrum. These "source experiments" can be performed with concentrations of ${ }^{57} \mathrm{Co}$ in the ppm range, providing a potent local probe in the material. Another advantage of source experiments is that the samples are usually so dilute in the Mössbauer isotope that there is no thickness distortion of the measured spectrum. The single-line absorber, typically sodium ferrocyanide containing $0.2 \mathrm{mg} / \mathrm{cm}^{2}$ of ${ }^{57} \mathrm{Fe}$, may itself have thickness distortion, but it is the same for all Doppler velocities. The net effect of absorber thickness is a broadening of spectral features without a distortion of intensities.

\section{Synchrotron Sources}

Since 1985 (Gerdau et al., 1985), it has become increasingly practical to perform Mössbauer spectrometry measurements with a synchrotron source of radiation, rather than a radioisotope source. This work has become more routine with the advent of Mössbauer beamlines at the European Synchrotron Radiation Facility at Grenoble, France, the Advanced Photon Source at Argonne National Laboratory, Argonne, Illinois, and the SPring- 8 facility in Harima, Japan. Work at these facilities first requires success in an experiment approval process. Successful beamtime proposals will not involve experiments that can be done with radioisotope sources. Special capabilities that are offered by synchrotron radiation sources are the time structure of the incident radiation, its brightness and collimation, and the prospect of measuring energy spectra off-resonance to study phonons and other excitations in solids.

Synchrotron radiation for Mössbauer spectrometry is provided by an undulator magnet device inserted in the synchrotron storage ring. The undulator has tens of magnetic poles, positioned precisely so that the electron accelerations in each pole are arranged to add in phase. This provides a high concentration of radiation within a narrow range of angle, somewhat like Bragg diffraction from a crystal. Synchrotron sources offer extremely high brightness because they are good approximations to point 
sources of radiation. As such, they are amenable to focusing with $\mathrm{x}$-ray mirrors or zone plates, and photon beams of a few microns diameter are obtained today. These tight beams can be used to advantage in measurements on materials in extreme environments, which tend to have very small volumes. Synchrotron Mössbauer spectrometry has become an important technique for studying electronic and dynamic properties of materials in diamond anvil cells, where high pressures an high temperatures are achieved in tiny volumes.

Measurements of energy spectra are usually impractical with a synchrotron source, but equivalent spectroscopic information is available in the time domain. The method may be understood as "Fourier transform Mössbauer spectrometry." A synchrotron flash, with time coherence less than $1 \mathrm{~ns}$, first excites all resonant nuclei in the sample. Over the period of time for nuclear decay, $100 \mathrm{~ns}$ or so, the nuclei emit photon waves with energies characteristic of their hyperfine fields. Assume that there are two such hvnerfine fields in the solid, providing photons of energy $E_{\gamma}^{0}+\varepsilon_{1}$ and $E_{\gamma}^{0}+\varepsilon_{2}$. In the forward scattering direction, the two photon waves can add in phase. The time dependence of the photon at the detector is obtained by the coherent sum as in Equation 3

$$
T(t)=\mathrm{e}^{\mathrm{i}\left(E_{\gamma}^{0}+\varepsilon_{1}\right) t / \hbar}+\mathrm{e}^{\mathrm{i}\left(E_{\gamma}^{0}+\varepsilon_{2}\right) t / \hbar}
$$

The photon intensity at the detector, $I(t)$, has the time dependence

$$
I(t)=T^{*}(t) T(t)=2\left\{1+\cos \left[\left(\varepsilon_{2}-\varepsilon_{1}\right) t / \hbar\right]\right\}
$$

When the energy difference between levels, $\varepsilon_{2}-\varepsilon_{1}$, is greater than the natural linewidth, $\Gamma$, the forward scattered intensity measured at the detector will undergo a number of oscillations during the time of the nuclear decay. These "quantum beats" can be Fourier transformed to provide energy differences between hyperfine levels of the nucleus (Smirnov, 1996). It should be mentioned that forward scattering from thick samples also shows a phenomenon of "dynamical beats," which involve energy interchanges between scattering processes. Untangling the quantum beats from the dynamical beats is usually done by fitting a sophisticated physics model to the experimental data (Sturhahn and Gerdau, 1994).

\section{Valence and Spin Determination}

The isomer shift, with supplementary information provided by the quadrupole splitting, often can be used to determine the valence and spin of ${ }^{57} \mathrm{Fe}$ and ${ }^{119} \mathrm{Sn}$ atoms. The isomer shift is proportional to the electron density at the nucleus, but this is influenced by the different $\sigma$ - and $\pi$-donor acceptance strengths of surrounding ligands, their electronegativities, covalency effects, electronic screening, and other phenomena. It is usually best to have some independent knowledge about the electronic state of $\mathrm{Fe}$ or $\mathrm{Sn}$ in the material before attempting to determine valence. Nevertheless, even for unknown materials, valence and spin can often be determined reliably for the Mössbauer isotope.

It is sometimes possible to use isomer shifts (IS) to find the number of $4 s$ and $3 d$ electrons at an $\mathrm{Fe}$ atom. This requires calibration curves. For ${ }^{57} \mathrm{Fe}$, these are plots of the IS versus the number of $4 s$ electrons at the iron atom. These plots do not consist of just one curve, however. The $3 d$ electrons screen the $4 s$ electrons from the nucleus, and with more $3 d$ electrons on the $\mathrm{Fe}$ atom, there is a more shallow slope of IS vs. $4 s$ count. For example, a set of three curves for $3 d^{5} 4 s^{\mathrm{x}}, 3 d^{6} 4 s^{\mathrm{x}}$, and $3 d^{7} 4 s^{\mathrm{x}}$ have slopes of approximately $(+4.0 \mathrm{~mm} / \mathrm{s}) /(4 \mathrm{~s}$ electron $),(+3.0 \mathrm{~mm} / \mathrm{s}) /(4 s$ electron $)$, and $(+1.4 \mathrm{~mm} / \mathrm{s}) /(4 s$ electron), respectively (Walker et al., 1961). (As an example, a change of $\Delta x=+0.3$ for a $3 d^{5} 4 s^{\mathrm{x}}$ configuration will add a positive isomer shift of +1.2 $\mathrm{mm} / \mathrm{s}$.) These three curves are offset by the effects of $3 d$ electrons on the other $s$ electrons at the ${ }^{57} \mathrm{Fe}$ nucleus. The isomer shifts for zero $4 s$ electrons are $+0.6,+1.4$ and +1.6 $\mathrm{mm} / \mathrm{s}$ for $3 d^{5} 4 s^{0}, 3 d^{6} 4 s^{0}$, and $3 d^{7} 4 s^{0}$, respectively (these shifts are with respect to stainless steel). If the number of $4 s$ electrons is known, the isomer shift can therefore be used to obtain the number of $3 d$ electrons at the $\mathrm{Fe}$ atom. Obtaining both $4 s$ and $3 d$ electron counts from a single isomer shift measurement is, of course, not possible in general, but there are ranges of isomer shifts that are expected for different valence states of Fe.

The ${ }^{57} \mathrm{Fe}$ isomer shifts shown in Figure 4 are useful for determining the valence and spin state of $\mathrm{Fe}$ ions. If the ${ }^{57} \mathrm{Fe}$ isomer shift of an unknown compound is $+1.2 \mathrm{~mm} / \mathrm{s}$ with respect to bcc Fe, for example, it is identified as highspin $\mathrm{Fe}(\mathrm{II})$. Low-spin $\mathrm{Fe}(\mathrm{II})$ and $\mathrm{Fe}(\mathrm{III})$ compounds show very similar isomer shifts, so it is not possible to distinguish between them on the basis of isomer shift alone. Fortunately, there are distinct differences in the electric quadrupole splittings of these electronic states. For low spin $\mathrm{Fe}(\mathrm{II})$, the quadrupole splittings are rather small, being in the range of 0 to $0.8 \mathrm{~mm} / \mathrm{s}$. For low spin Fe(III) the electric quadrupole splittings are larger, being in the range 0.7 to $1.7 \mathrm{~mm} / \mathrm{s}$. The other oxidation states shown in Figure 4 are not so common, and tend to be of greater interest to chemists than materials scientists.

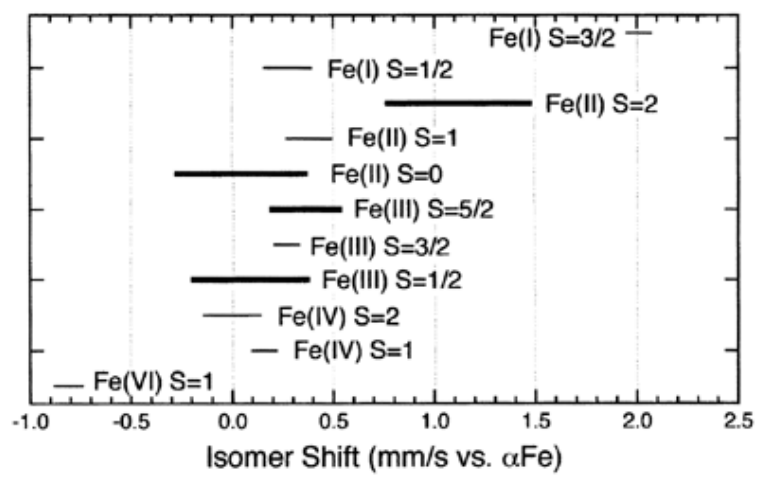

Figure 4. Ranges of isomer shifts in Fe compounds with various valences and spin states, with reference to bcc Fe metal at $300 \mathrm{~K}$. Thicker lines are more common configurations (Greenwood and Gibb, 1971; Gütlich, 1975).

The electric quadrupole splitting (EQS) is less directly interpretable in terms of the electronic state of Fe atoms (at least compared to the isomer shift). Nevertheless, the EQS is often large, easy to measure, and helpful for showing if there is more than one electronic environment for Fe atoms 
in a material. Equation 22 shows that the EQS is proportional to the electric field gradient (EFG), so the interpretation of the measured EQS involves relating the electronic state of the Fe to the symmetry of its electronic environment. For ${ }^{57} \mathrm{Fe}$ we develop this relationship in two steps: 1) the effect of the local chemical environment on the electronic levels of the valence electrons, using crystal field theory, and 2) the effect of the asymmetry of the grund state charge distribution on the EFG at the ${ }^{57} \mathrm{Fe}$ nucleus.

First, the $3 d$ atomic orbitals have different shapes that have different bond energies when placed on crystal sites. For two Fe atoms on neighboring crystal sites, the different lobes of the $3 d$ orbitals point towards or away from each other, depending on the crystal structure and shape of the orbitals $\left(3 z^{2}-r^{2}, x^{2}-y^{2}, x z, y x, x y\right)$. Two important local configurations are tetrahedral and octahedral environments around the $\mathrm{Fe}$ atom. In a tetrahedral environment, the $3 z^{2}$ $r^{2}, x^{2}-y^{2}$ orbitals (called $e$ ) have better bonding overlaps than the $x z, y x, x y$ (called $t_{2}$ ). For an octahedral environment, however, the $x z, y x, x y$ (called $t_{2 g}$ ) will make better bonds than the $3 z^{2}-r^{2}, x^{2}-y^{2}$ levels (called $e_{g}$ ).

The second step is to use the ground state for the occupancy of these orbitals to calculate the electric field gradient from the charge distribution. A simple approach is to use a point charge model, ignoring complexities of screening, for example. (This model often works better than it deserves.) This model gives symmetrical electric field distributions at a central ${ }^{57} \mathrm{Fe}$ atom, and no EFG for pure tetrahedral and octahedral environments when all five types of $3 d$-orbitals are occupied. This is the case for high spin $\mathrm{Fe}(\mathrm{III})$, which has five $3 d$ electrons in each of the orbitals $\left(3 z^{2}-r^{2}, x^{2}-y^{2}, x z, y x, x y\right)$, and in fact that $\mathrm{Fe}(\mathrm{III})$ compounds with spins of $5 / 2$, tend to have small EQS in Mössbauer spectra. Some EQS is expected, however, when there is a distortion of the symmetry of the local environment of the surrounding atoms.

The $\mathrm{Fe}(\mathrm{II})$ ions have an extra electron, so at least one of the five $d$-orbitals $\left(3 z^{2}-r^{2}, x^{2}-y^{2}, x z, y x, x y\right)$ contains two electrons of opposite spin. The additional $3 d$ electron will select among the lower energy states in the crystal field, such as the $t_{2 g}$ state when the Fe atom is in an octahedral environment (giving a $t_{2 g}{ }^{4} e_{g}{ }^{2}$ configuration). This is the high spin $\mathrm{Fe}(\mathrm{II})$ configuration, for which the extra electron compared to $\mathrm{Fe}(\mathrm{III})$ tends to cause a large EQS. Usually a smaller EQS is found for the low spin configuration of $\mathrm{Fe}(\mathrm{II})$ that occurs when the octahedral crystal field is strong (so the large crystal field splitting energy exceeds the exchange interaction that tends to align the spins). The low spin $\mathrm{Fe}(\mathrm{II})$ has a configuration of $t_{2 g}{ }^{6} e_{g}{ }^{0}$ in an octahedral environment.

Sometimes the differences in the energy levels for the $3 d$ electrons are modest, and it is possible for the populations of the levels to change with temperature, pressure, or chemical composition. "Spin transitions" in $\mathrm{Fe}$ (II) compounds, where the balance of low-spin and highspin states undergo a change, have been studied by Mössbauer spectrometry. Besides the populations of spins, set by Boltzmann factors for the energy level differences and $k_{\mathrm{B}} T$, it is also possible to observe fluctuations in the EFG owing to the paired electron in Fe(II) moving between the different states available to it. Even if the levels are the same, there can be a change in direction of the EFG, and this might occur on the scale of the Mössbauer measurement time.

Mixed-valent compounds are particularly interesting for study by Mössbauer spectrometry. Figure 5 presents spectra at various temperatures of a solid solution of $\mathrm{Li}_{0.6} \mathrm{FePO}_{4}$ with the olivine crystal structure. At low temperatures the $\mathrm{Fe}(\mathrm{III})$ and and $\mathrm{Fe}(\mathrm{II})$ ions both show quadrupole doublets, although there is overlap of two of their peaks at left. (For each doublet, however, we know that areas of the two peaks must be equal for a powder sample.) At $25^{\circ} \mathrm{C}$ the centroid of the $\mathrm{Fe}(\mathrm{III})$ is shifted to the left of the centroid for $\mathrm{Fe}(\mathrm{II})$; $\mathrm{Fe}(\mathrm{II})$ has the more positive isomer shift. At elevated temperature two effects are seen. First, there is a collapse of the EQS, where the Fe(III) and especially the $\mathrm{Fe}(\mathrm{II})$ doublet show a decreased splitting with temperature. Second, there is a positive shift of the Fe(III) spectrum, but a negative shift of the Fe(II) spectrum. The isomer shifts of $\mathrm{Fe}(\mathrm{III})$ and $\mathrm{Fe}(\mathrm{II})$ merge towards each other with increased temperature. These changes in IS and EQS originate with the dynamical process of small polaron hopping in $\mathrm{Li}_{\mathrm{x}} \mathrm{FePO}_{4}$. Polarons comprise the Fe ion, together with its distorted neighborhood. Moving an electron from an $\mathrm{Fe}(\mathrm{II})$ site to an adjacent $\mathrm{Fe}$ (III) site requires changing the positions of neighboring oxygen and phosphorous atoms, and thermal activation is required for this process. Small polaron hopping is the mechanism of electrical conductivity of $\mathrm{Li}_{0.6} \mathrm{FePO}_{4}$, and is important for when this material is used for electrodes in rechargeable batteries. When polaron hopping occurs at a rate of $10^{8} \mathrm{~Hz}$, Mössbauer spectrometry no longer sees unique spectral signatures for $\mathrm{Fe}(\mathrm{II})$ and $\mathrm{Fe}(\mathrm{III})$, either for their IS or EQS.

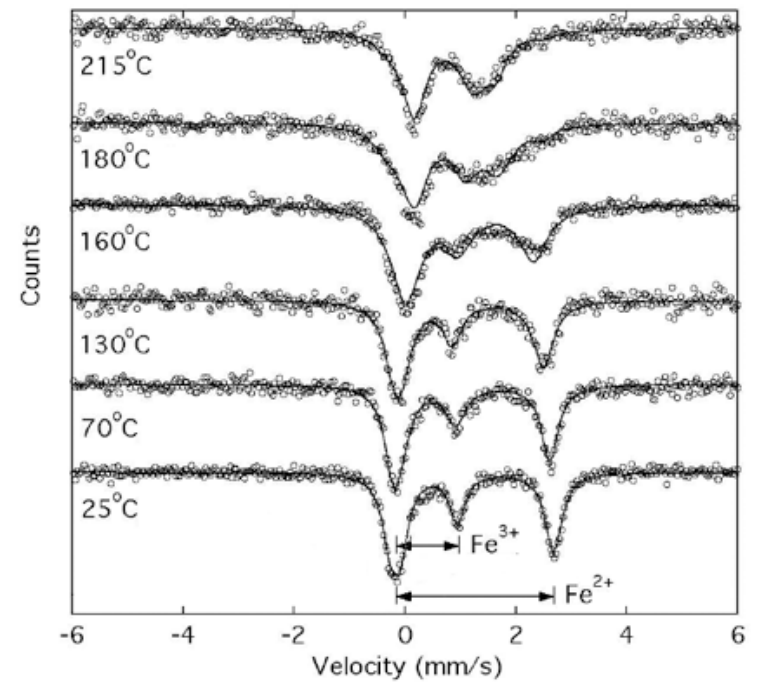

Figure 5. Mössbauer spectra of disordered solid solution of $\mathrm{Li}_{0.6} \mathrm{FePO}_{4}$ with the olivine structure, acquired in-situ at elevated temperatures (Dodd et al., 2007). The merger of the two doublets occurs when the charge hopping frequency is of order $10^{8} \mathrm{~Hz}$ or faster.

The previous analysis of valence and spin state assumed that the material is not magnetically ordered. In cases where a hyperfine magnetic field is present, identification of the chemical state of $\mathrm{Fe}$ is sometimes even easier. Table 1 presents a few examples of hyperfine 
magnetic fields and isomer shifts for common magnetic oxides and oxyhydroxides (Simmons and Leidheiser, 1976). This table is given as a convenient guide, but the hyperfine parameters may depend on crystalline quality and stoichiometry (Bowen et al.,1993).

Table 1. Hyperfine Parameters of Common Oxides and Oxyhydroxides

\begin{tabular}{lcccc} 
Compound (Fe Site) & HMF (T) & Q.S. & I.S. (vs. Fe) & Temp. (K) \\
\hline$\alpha-\mathrm{FeOOH}$ & 50.0 & -0.25 & & 77 \\
$\alpha-\mathrm{FeOOH}$ & 38.2 & -0.25 & +0.61 & 300 \\
$\beta-\mathrm{FeOOH}$ & 48.5 & 0.64 & +0.38 & 80 \\
$\beta-\mathrm{FeOOH}$ & 0 & 0.62 & +0.39 & 300 \\
$\gamma$-FeOOH & 0 & 0.60 & +0.38 & 295 \\
$\delta$-FeOOH (big xtls.) & 42.0 & & +0.35 & 295 \\
$\mathrm{FeO}$ & & 0.8 & +0.93 & 295 \\
$\mathrm{Fe}_{3} \mathrm{O}_{4}(\mathrm{Fe}(\mathrm{III}), \mathrm{A})$ & 49.3 & & +0.26 & 298 \\
$\mathrm{Fe}_{3} \mathrm{O}_{4}(\mathrm{Fe}(\mathrm{II}, \mathrm{III}), \mathrm{B})$ & 46.0 & & +0.67 & 298 \\
$\alpha-\mathrm{Fe}_{2} \mathrm{O}_{3}$ & 51.8 & +0.42 & +0.39 & 296 \\
$\gamma-\mathrm{Fe}_{2} \mathrm{O}_{3}(\mathrm{~A})$ & 50.2 & & +0.18 & 300 \\
$\gamma-\mathrm{Fe}_{2} \mathrm{O}_{3}(\mathrm{~B})$ & 50.3 & & +0.40 & 300 \\
\hline
\end{tabular}

${ }^{a}$ Abbreviations: HMF, hyperfine magnetic field; I.S., isomer shift; Q.S., quadrupole splitting; T, tesla.

The isomer shifts for ${ }^{119} \mathrm{Sn}$ compounds have a wider range than for ${ }^{57} \mathrm{Fe}$ compounds. Isomer shifts for compounds with $\mathrm{Sn}(\mathrm{IV})$ ions have a range from -0.5 to $+1.5 \mathrm{~mm} / \mathrm{s}$ versus $\mathrm{SnO}_{2}$. For $\mathrm{Sn}$ (II) compounds, the range of isomer shifts is +2.2 to +4.2 versus $\mathrm{SnO}_{2}$. Within these ranges it is possible to identify other chemical trends. In particular, for Sn compounds there is a strong correlation of isomer shift with the electronegativity of the ligands. This correlation between isomer shift and ligand electronegativity is especially reliable for $\mathrm{Sn}(\mathrm{IV})$ ions. Within a family of $\mathrm{Sn}(\mathrm{IV})$ compounds of similar coordination, the isomer shift depends on the electronegativity of the surrounding ligands as $-1.27 \chi$ $\mathrm{mm} / \mathrm{s}$, where $\chi$ is the Pauling electronegativity. The correlation with $\mathrm{Sn}(\mathrm{II})$ is less reliable, in part because of the different coordinations found for this ion.

Finally, it should be mentioned that there have been a number of efforts to correlate the local coordination of ${ }^{57} \mathrm{Fe}$ with the electric quadrupole splitting. These correlations are often reliable within a specific class of compounds, typically showing a semiquantitative relationship between quadrupole splitting and the degree of distortion of the local atomic structure.

\section{Phase Analysis}

When more than one crystallographic phase is present in a material containing ${ }^{57} \mathrm{Fe}$ or ${ }^{119} \mathrm{Sn}$, it is often possible to determine the phase fractions at least semiquantitatively. Usually some supplemental information is required before quantitative information can be derived. For example, most multiphase materials contain several chemical elements. Since Mössbauer spectrometry detects only the Mössbauer isotope, to determine the volume fraction of each phase, it is necessary to know its concentration of Mössbauer isotope. Quantitative phase analysis tends to be most reliable when the material is rich in the Mössbauer atom. Phase fractions in iron alloys, steels, and iron oxides can often be measured routinely by Mössbauer spectrometry (Schwartz, 1976; Simmons and Leidheiser, 1976; Cortie and Pollak, 1995; Campbell et al., 1995).

Mössbauer spectrometry is well suited for detecting small amounts of fcc phase in a bcc matrix, since the fcc phase is paramagnetic, and all its intensity appears as a single peak near the center of the spectrum. Amounts of fcc phase ("austenite") of $0.5 \%$ can be detected in iron alloys and steels, and quantitative analysis of the fcc phase fraction is straightforward. Figure 6 is an example of phase analysis of an Fe-Ni alloy, for which the interest was in determining the kinetics of fcc phase formation at $600^{\circ} \mathrm{C}$ (Fultz, 1982). The fcc phase, once formed, is stable at $500^{\circ} \mathrm{C}$ but not at room temperature. To determine the amount of fcc phase formed at $600^{\circ} \mathrm{C}$ it was necessary to measure Mössbauer spectra at $500^{\circ} \mathrm{C}$ without an intervening cooling to room temperature for spectrum acquisition.

The spectra in Figure 6 clearly show the six-line pattern of the bcc phase and the growth of the single peak at -0.4 $\mathrm{mm} / \mathrm{s}$ from the fcc phase. These spectra a show three other features that are common to many Mössbauer spectra. First, the spectrum at the top of Figure 6 shows a broadening of the outer lines of the sextet with respect to the inner lines (also see Fig. 2). This broadening originates with a distribution of hyperfine magnetic fields in alloys. The different numbers of Ni neighbors about the various ${ }^{57} \mathrm{Fe}$ atoms in the bcc phase cause different perturbations of the ${ }^{57} \mathrm{Fe}$ HMF. Second, the Curie temperature of bcc $\mathrm{Fe}-8.9$ at. $\% \mathrm{Ni}$ is $\sim 700^{\circ} \mathrm{C}$. At the Curie temperature the average lattice magnetization is zero, and the HMF is also zero. At $500^{\circ} \mathrm{C}$ the alloy is approaching the Curie temperature, and shows a strong reduction in its HMF as evidenced by the smaller splitting of the six line pattern with respect to the pattern at $23^{\circ} \mathrm{C}$. Finally, at $500^{\circ} \mathrm{C}$ the entire spectrum is shifted to the left towards more negative isomer shift. This is the relativistic thermal shift of Equation 20.

To obtain the phase fractions, the fcc and bcc components of the spectrum were isolated and integrated numerically. Isolating the fcc peak was possible by digital subtraction of the initial spectrum from spectra measured after different annealing times. The fraction of the fcc spectral component then needed two correction factors to convert it into a molar phase fraction. One factor accounted for the different chemical compositions of the fcc and bcc phases (the fee phase was enriched in Ni to about 25\%). A second factor accounted for the differences in recoil-free fraction of the two phases. Fortunately, the Debye temperatures of the two phases were known, and they differed little, so the differences in recoil-free fraction were not significant. The amount of fcc phase in the alloy at $500^{\circ} \mathrm{C}$ was found to change from $0.5 \%$ initially to $7.5 \%$ after $34 \mathrm{~h}$ of heating at $600^{\circ} \mathrm{C}$. 


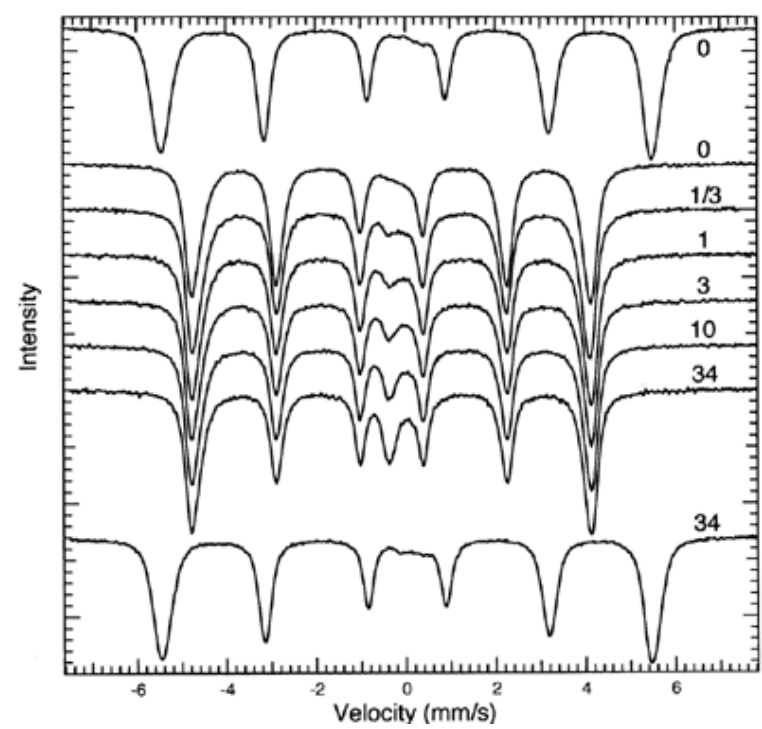

Figure 6. Mössbauer spectra of an alloy of $\mathrm{Fe}-8.9$ atomic $\% \mathrm{Ni}$. The initial state of the material was ferromagnetic bcc phase, shown by the six-line spectrum at the top of the figure. This top spectrum was acquired at $23^{\circ} \mathrm{C}$. The sample was heated in situ in the Mössbauer spectrometer to $600^{\circ} \mathrm{C}$, for the numbers of hours marked on the curves, to form increasing amounts of fcc phase, evident as the single paramagnetic peak near $-0.4 \mathrm{~mm} / \mathrm{s}$. This fee phase is stable at $500^{\circ} \mathrm{C}$, but not at $23^{\circ} \mathrm{C}$. so the middle spectra were acquired at $500^{\circ} \mathrm{C}$ in interludes between heatings at $600^{\circ} \mathrm{C}$ for various times. At the end of the high-temperature runs, the sample temperature was again reduced to $23^{\circ} \mathrm{C}$, and the final spectrum shown at the bottom of the figure showed that the fcc phase had transformed back into bcc phase. A trace of oxide is evident in all spectra as additional intensity around $+0.4 \mathrm{~mm} / \mathrm{s}$ at $23^{\circ} \mathrm{C}$.

\section{Solutes in bcc Fe Alloys}

The HMF in pure bcc $\mathrm{Fe}$ is $-33.0 \mathrm{~T}$ for every $\mathrm{Fe}$ atom, since every $\mathrm{Fe}$ atom has an identical chemical environment of $8 \mathrm{Fe}$ first-nearest-neighbors (1nn), $6 \mathrm{Fe} 2 \mathrm{nn}, 12 \mathrm{Fe} 3 \mathrm{nn}$, etc. In ferromagnetic alloys, however, the ${ }^{57} \mathrm{Fe} \mathrm{HMF}$ is perturbed significantly by the presence of neighboring solute atoms. In many cases, this perturbation is about +2.5 $\mathrm{T}$ (a reduction in the magnitude of the HMF) for each $1 \mathrm{nn}$ solute atom. A compilation of some HMF perturbations for $1 \mathrm{nn}$ solutes and $2 \mathrm{nn}$ solutes is presented in Figure 7. These data were obtained by analysis of Mössbauer spectra from dilute bcc Fe-X alloys (Vincze and Campbell, 1973; Vincze and Aldred, 1974; Fultz, 1993).

In general, the HMF perturbations at ${ }^{57} \mathrm{Fe}$ nuclei from nearest-neighbor solute atoms originate from several sources, but for nonmagnetic solutes such as $\mathrm{Si}$, the effects are fairly simple to understand. When the $\mathrm{Si}$ atom substitutes for an $\mathrm{Fe}$ atom in the bcc lattice, a magnetic moment of $2.2 \mu_{\mathrm{B}}$ is removed (the $\mathrm{Fe}$ ) and replaced with a magnetic hole (the $\mathrm{Si}$ ). The $4 s$ conduction electrons redistribute their spin density around the $\mathrm{Si}$ atom, and this redistribution is significant at $1 \mathrm{nn}$ and $2 \mathrm{nn}$ distances. The Fermi contact interaction and $H_{\text {eff }}$ (Equation 24) are sensitive to the $4 s$ electron spin density, which has finite probability at the ${ }^{57} \mathrm{Fe}$ nucleus. Another important feature of $3 p, 4 p$, and $5 p$ solutes is that their presence does not significantly affect the magnetic moments at neighboring $\mathrm{Fe}$ atoms. Bulk magnetization measurements on $\mathrm{Fe}-\mathrm{Si}$ and Fe-Al alloys, for example, show that the magnetic moment of the material decreases approximately in proportion to the fraction of $\mathrm{Al}$ or $\mathrm{Si}$ in the alloy. The core electron polarization, which involves exchange interactions between the unpaired $3 \mathrm{~d}$ electrons at the ${ }^{57} \mathrm{Fe}$ atom and its innershell s electrons, is therefore not much affected by the presence of Si neighbors. The dominant effect comes from the magnetic hole at the solute atom, which causes the redistribution of $4 \mathrm{~s}$ spin density. Figure 7 shows that the nonmagnetic $3 p, 4 p$, and $5 p$ elements all cause about the same HMF perturbation at neighboring ${ }^{57} \mathrm{Fe}$ atoms, as do the nonmagnetic early $3 d$ transition metals.

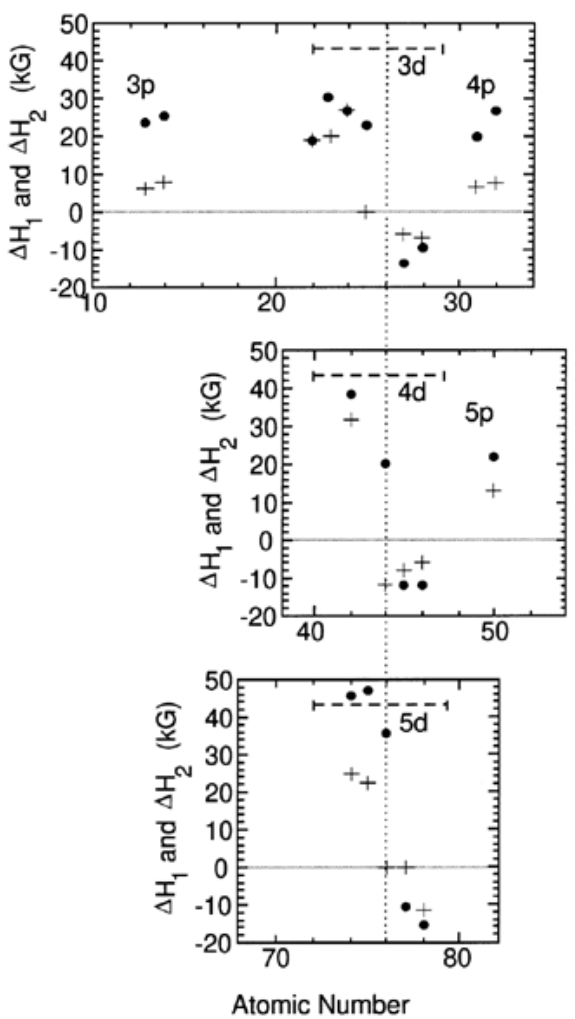

Figure 7. The hyperfine magnetic field perturbation, $\Delta H_{1}{ }^{X}$ (dots), at a Fe atom caused by one $1 \mathrm{nn}$ solute of type $\mathrm{X}$, and the $2 \mathrm{nn}$ perturbation, $\Delta H_{2}{ }^{X}$ (crosses) versus the atomic number of the solute. The vertical line denotes the column of $\mathrm{Fe}$ in the periodic table.

For solutes that perturb significantly the magnetic moments at neighboring $\mathrm{Fe}$ atoms, especially the late transition metals, the core polarization at the ${ }^{57} \mathrm{Fe}$ atom is altered. There is an additional complicating effect from the matrix $\mathrm{Fe}$ atoms near the resonant ${ }^{57} \mathrm{Fe}$ atom, whose magnetic moments are altered enough to affect the $4 s$ conduction electron polarization at the ${ }^{57} \mathrm{Fe}$ (Fultz, 1993).

The HMF distribution can sometimes provide detailed information on the arrangements of solutes in nondilute bcc Fe alloys. For most solutes (that do not perturb significantly the magnetic moments at $\mathrm{Fe}$ atoms), the $\mathrm{HMF}$ at a ${ }^{57} \mathrm{Fe}$ atom depends monotonically on the number of solute atoms 
in its $1 \mathrm{nn}$ and $2 \mathrm{nn}$ shells. Hyperfine magnetic field perturbations can therefore be used to measure the chemical composition or the chemical short-range order in an alloy containing up to 10 atomic \% solute or even more. In many cases, it is possible to distinguish among $\mathrm{Fe}$ atoms having different numbers of solute atoms as first neighbors, and then determine the fractions of these different first neighbor environments. This is considerably more information on chemical short-range order (SRO) than just the average number of solute neighbors, as provided by a $1 \mathrm{nn}$ WarrenCowley SRO parameter, for example.

An example of chemical short range order in an Fe-26 atomic \% $\mathrm{Al}$ alloy is presented in Figure 8. The material was cooled at a rate of $10^{6} \mathrm{~K} / \mathrm{s}$ from the melt by pistonanvil quenching, producing a polycrystalline ferromagnetic alloy with a nearly random distribution of $\mathrm{Al}$ atoms on the bcc lattice. With low-temperature annealing, the material evolved toward its equilibrium state of $\mathrm{D}_{3}$ chemical order. The Mössbauer spectra in Figure 8A change significantly as the alloy evolves chemical order. The overlap of several sets of six line patterns does confuse the physical picture, however, and further analysis requires the extraction of an HMF distribution from the experimental data. Software packages available for such work are described below (see Data Analysis). Figure 8B shows HMF distributions extracted from the three spectra of Figure 8A. At the top of Figure $8 \mathrm{~B}$ are markers indicating the numbers of $\mathrm{Al}$ atoms in the $1 \mathrm{nn}$ shell of the ${ }^{57} \mathrm{Fe}$ nucleus associated with the HMF. With low-temperature annealing, there is a clear increase in the numbers of ${ }^{57} \mathrm{Fe}$ atoms with 0 and $4 \mathrm{~A} 1$ neighbors, as expected when $\mathrm{D}_{3}$ order is evolving in the material. The perfectly ordered $\mathrm{D}_{3}$ structure has two chemical sites for $\mathrm{Fe}$ atoms, one with $0 \mathrm{~A} 1$ neighbors and the other with 4 A1 neighbors, in a 1:2 ratio. The HMF distributions were fit to a set of Gaussian functions to provide data on the chemical short range order in the alloys. These data on chemical short-range order are presented in Figure 8C.

\section{Crystal Defects and Nano-Particles}

Since Mössbauer spectrometry probes local environments around a nucleus, it has often been proposed that Mössbauer spectra should be sensitive to the local atomic structures at grain boundaries and defects such as dislocations and vacancies. This is in fact true, but the measured spectra are an average over all Mössbauer atoms in a sample. Unless the material is chosen carefully so that the Mössbauer atom is segregated to the defect of interest, the spectral contribution from the defects is usually overwhelmed by the contribution from Mössbauer atoms in regions of perfect crystal.

The interest in nanocrystalline materials, however, has provided a number of new opportunities for Mössbauer spectrometry (Herr et al., 1987; Fultz et al., 1995). The number of atoms and near grain boundaries in nanocrystals is typically $35 \%$ for bcc Fe alloys with crystallite sizes of 7 $\mathrm{nm}$ or so. Such a large fraction of grain boundary atoms makes it possible to identify distinct contributions from Mössbauer atoms at grain boundaries, and to identify their local electronic environment. When performing such studies on a new nanomaterial, it is usually important to measure Mössbauer spectra for samples of different nanocrystalline sizes, $l$. If the spectrum has a component with an areal fraction that scales as $1 / l$, this component is a candidate for $\mathrm{Fe}$ atoms in grain boundaries and at crystal surfaces. It is also important to check that this areal fraction is consistent with the expected fraction of ${ }^{57} \mathrm{Fe}$ atoms in these locations.
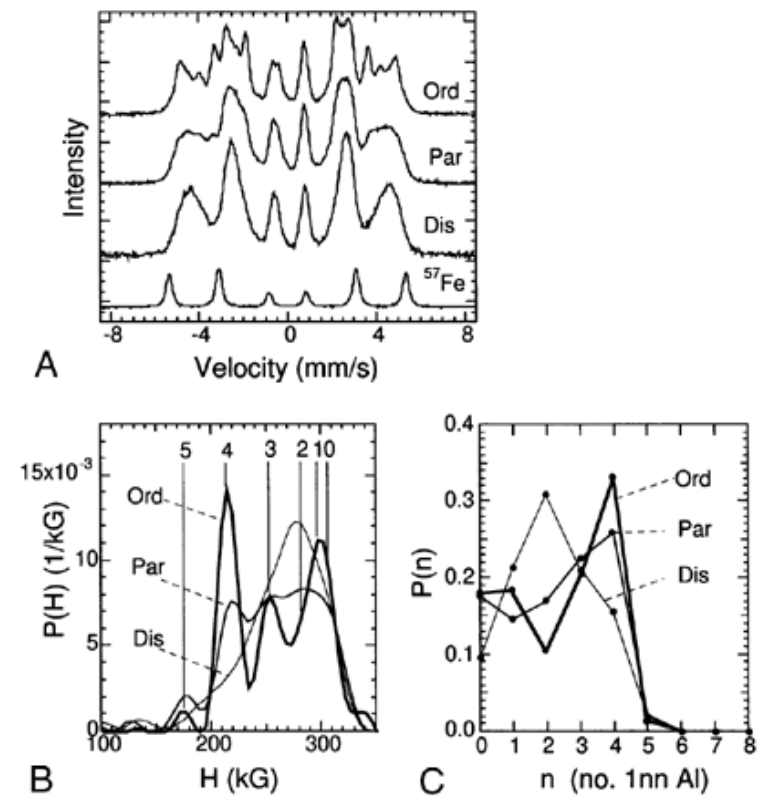

Figure 8. (A) Conversion electron Mössbauer spectra from a specimen of bcc ${ }^{57} \mathrm{Fe}$ and three specimens of disordered, partially ordered, and $\mathrm{DO}_{3}$-ordered ${ }^{57} \mathrm{Fe}_{3} \mathrm{Al}$. (B) HMF distribution of the ${ }^{57} \mathrm{Fe}_{3} \mathrm{Al}$ specimens. Peaks in the HMF distribution are labeled with numbers indicating the different numbers of $1 \mathrm{nn}$ Al neighbors about the ${ }^{57} \mathrm{Fe}$ atom. (C) Probabilities for the ${ }^{57} \mathrm{Fe}$ atom having various numbers, $n$, of $\mathrm{Al}$ atoms as $1 \mathrm{nn}$.

Mössbauer spectrometry can provide detailed information on some features of small-particle magnetism (Mørup, 1990). When a magnetically ordered material is in the form of a very small particle, it is easier for thermal energy to realign the direction of its magnetization. The particle retains its magnetic order, but the change in axis of magnetization will disturb the shape of the Mössbauer spectrum if the magnetic realignment occurs on the time scale $t_{\mathrm{s}}$, which is $\hbar$ divided by the hyperfine magnetic field energy (see Relaxation Phenomena for discussion of the time window for measuring hyperfine interactions). An activation energy is associated with this "superparamagnetic" behavior, which is the magnetocrystalline anisotropy energy times the volume of the crystallite, $\kappa \mathrm{V}$. The probability of activating a spin rotation in a small particle is the Boltzmann factor for overcoming the anisotropy energy, so the condition for observing a strong relaxation effect in the Mössbauer spectrum is

$$
t_{\mathrm{s}}=A \exp \left(-\kappa \mathrm{V} / k_{\mathrm{B}} T_{\mathrm{b}}\right)
$$


The temperature, $T_{\mathrm{b}}$, satisfying Equation 28 is known as the "blocking temperature." The prefactor of Equation 28, the attempt frequency, is not so well understood, so studies of superparamagnetic behavior often study the blocking temperature versus the volume of the particles. In practice, most clusters of small particles have a distribution of blocking temperatures, and there are often interactions between the magnetic moments at adjacent particles. These effects can produce Mössbauer spectra with a wide variety of shapes, including very broad Lorentizian lines.

At temperatures below $T_{\mathrm{b}}$, the magnetic moments of small particles undergo small fluctuations in their alignment. These small-amplitude fluctuations can be considered as vibrations of the particle magnetization about an average orientation, which serve to reduce the HMF by a modest amount. At increasing temperatures around $T_{\mathrm{b}}$, however, large fluctuations occur in the magnetic alignment. The result is first a severe uncertainty of the HMF distribution, leading to a very broad background in the spectrum, followed by the growth of a paramagnetic peak near zero velocity. All of these effects can be observed in the spectra shown in Figure 9. Here, the biomaterial samples comprised a core of haemosiderin, an iron storage compound, encapsulated within a protein shell. A clear six-line pattern is observed at $4.2 \mathrm{~K}$, but the splitting of these six lines is found to decrease with temperature owing to small amplitude fluctuations in magnetic alignment. At temperatures around 40 to $70 \mathrm{~K}$, a broad background appears under the measured spectrum, and a paramagnetic doublet begins to grow in intensity with increasing temperature. These effects are caused by large thermal reorientations of the magnetization. Finally, by 200 $\mathrm{K}$, the thermally induced magnetic fluctuations are all of large amplitude and of characteristic times too short to permit a HMF to be detected by Mössbauer spectrometry.

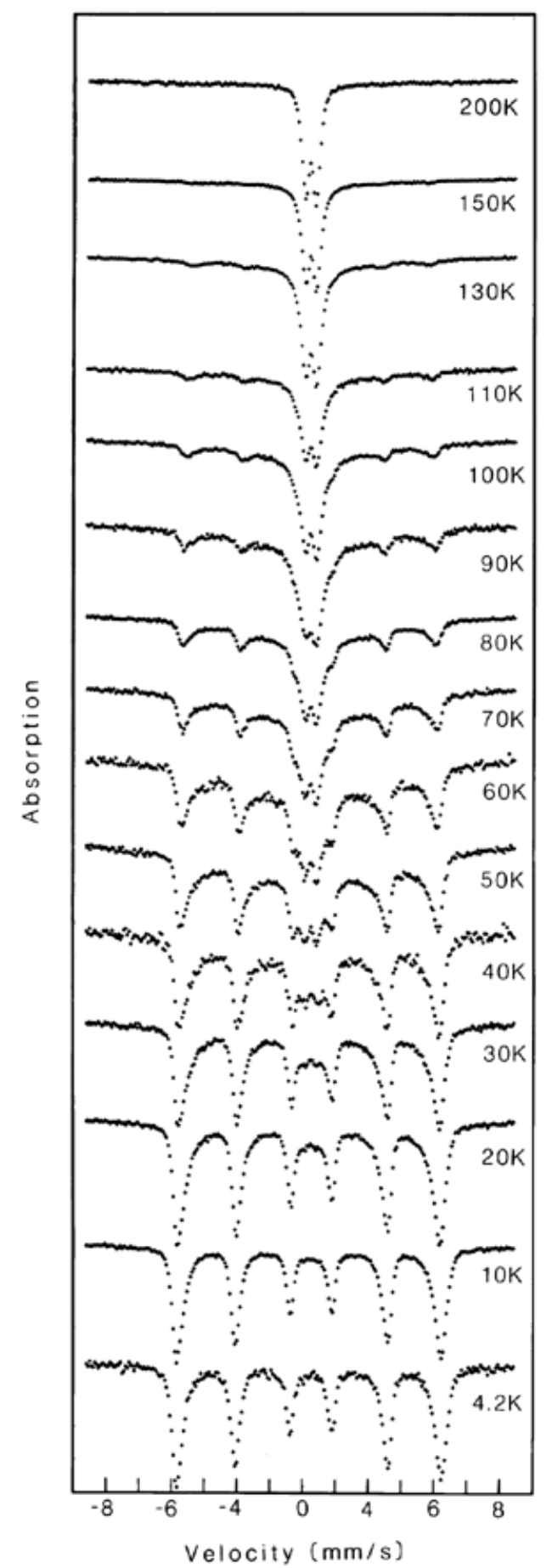

Figure 9. Mössbauer spectra from a specimen of haemosiderin, showing the effects of superparamagnetism with increasing temperature (Bell et al., 1984; Dickson, 1987). 


\section{DATA ANALYSIS AND INITIAL INTERPRETATION}

Mössbauer spectra are often presented for publication with little or no processing. An obvious correction that can be applied to most transmission spectra is a correction for thickness distortion (see Sample Preparation). This correction is rarely performed, however, in large part because the thickness of the specimen is usually not known or the thickness is not uniform. The specimen is typically prepared to be thin, or at least this is assumed, and the spectrum is assumed to be representative of the Mössbauer absorption cross-section.

A typical goal of data analysis is to find individual hyperfine parameters, or more typically a distribution of hyperfine parameters, that characterize a measured spectrum. For example, the HMF distribution of Figure 2 should resemble a delta function centered at $330 \mathrm{kG}$. On the other hand, the HMF distribution of Figure 8B shows a number of peaks that are characteristic of different local chemical environments. Distributions of electric quadrupole splittings and isomer shifts are also useful for understanding heterogeneities in the local atomic arrangements in materials.

Several software packages are available to extract distributions of hyperfine parameters from Mössbauer spectra (Hesse and Rutbartsch, 1974; Le Car and DuBoise, 1979; Brand and Le Caër, 1988; Lagarec and Rancourt, 1997). These programs are often distributed by their authors who may be located with the Mössbauer Information eXchange (see Internet Resources). The different programs extract hyperfine distributions from experimental spectra with different numerical approaches, but all will show how successfully the hyperfine distribution can be used to regenerate the experimental spectrum.

In the presence of statistical noise, the reliability of these derived hyperfine distributions must be considered carefully. In particular, over small ranges of hyperfine parameters, the hyperfine distributions are not unique. For example, it may be unrealistic to distinguish one Lorentzian-shaped peak centered at a particular velocity from the sum of several peaks distributed within a quarter of a linewidth around this same velocity. This nonuniqueness can lead to numerical problems in extracting hyperfine distributions from experimental data. Some software packages use smoothing parameters to penalize the algorithm when it picks a candidate HMF distribution with sharp curvature. When differences in hyperfine distributions are small, there is always an issue of their uniqueness. Sometimes the data analysis cannot distinguish between different types of hyperfine distributions. For example, a spectrum that has been broadened by an EFG distribution, or even an HMF distribution, can be fit perfectly with an IS distribution. The physical origin of hyperfine distributions may not be obvious, especially when the spectrum shows little structure. Application of an external magnetic field may be helpful in identifying a weak HMF, however.

In general, distributions of all three hyperfine parameters (IS, EFG, HMF) will be present simultaneously in a measured spectrum. These parameters may be correlated; for example nuclei having the largest HMF may have the largest (or smallest) IS. Sorting out these correlations is often a research topic in itself, although the software for calculating hyperfine distributions typically allows for simple linear correlations between the distributions.

Both the EFG and the HMF use an axis of quantization for the nuclear spin. However, the direction of magnetization (for the HMF axis) generally does not coincide with the directions of the chemical bonds responsible for the EFG. The general case with comparable hyperfine interaction energies of HMFs and EFGs is quite complicated, and is well beyond the scope of this unit. Some software packages using model spin Hamiltonians are available to calculate spectra acquired under these conditions, however. In the common case when the HMF causes much larger spectral splittings than the EFG, with polycrystalline samples the usual effect of the EFG is a simple broadening of the peaks in the magnetic sextet, with no shifts in their positions.

With even modest experimental care, Mössbauer spectra can be highly reproducible from run to run. For example, the Mössbauer spectrum in Figure 2 was repeated many times over a time period of several years. Almost all of these bcc Fe spectra had data points that overlaid on top of each other, point by point, within the accuracy of the counting statistics. Because of this reproducibility, it is tempting and often appropriate to try to identify spectral features with energy width smaller than the characteristic linewidth. An underexploited technique for data analysis is "partial deconvolution" or "thinning." Since the lineshape of each nuclear transition is close to a Lorentzian function, and can be quite reproducible, it is appropriate to deconvolute a Lorentzian function from the experimental spectrum. This is the same algorithm as for obtaining an IS distribution, but no assumptions about the origin of the hyperfine distributions are implied by the thinning process. The net effect is to sharpen the peaks from the experimental Mössbauer spectrum, and this improvement in effective resolution can be advantageous when overlapping peaks are present in the spectra. The method does require excellent counting statistics to be reliable, however.

Finally, in spite of all the ready availability of computing resources, it is always important to look at differences in the experimental spectra themselves. Sometimes a digital subtraction of one normalized spectrum from another is an excellent way to identify changes in a material. As a general rule, if no differences are detected by direct inspection of the data, changes in the hyperfine distributions obtained by computer software should not be believed. For this reason it is still necessary to show actual experimental spectra in research papers that present results from Mössbauer spectrometry.

\section{SAMPLE PREPARATION}

A central concern for transmission Mössbauer spectrometry is the choice and control of specimen thickness. The natural thickness of a specimen is $t$

$$
t \equiv\left(f_{\mathrm{a}} n_{\mathrm{a}} \sigma_{\mathrm{a}}\right)^{-1}
$$


where $f_{\mathrm{a}}$ is the recoil-free fraction of the Mössbauer isotope in the specimen, $n_{\mathrm{a}}$ is the number of Mössbauer nuclei per $\mathrm{cm}^{3}$, and $\sigma_{\mathrm{a}}$ is the cross-section in units of $\mathrm{cm}^{2}$. The $f_{\mathrm{a}}$ can be estimated from Equation 19, for which it is useful to know that the $\gamma$-ray energies are $14.41 \mathrm{keV}$ for ${ }^{57} \mathrm{Fe}, 23.875$ $\mathrm{keV}$ for ${ }^{119} \mathrm{Sn}$, and $21.64 \mathrm{keV}$ for ${ }^{151} \mathrm{Eu}$. To obtain $n_{\mathrm{a}}$ it is important to know that the natural isotopic abundance is $2.2 \%$ for ${ }^{57} \mathrm{Fe}, 8.6 \%$ for ${ }^{119} \mathrm{Sn}$, and $48 \%$ for ${ }^{151} \mathrm{Eu}$. The cross-sections for these isotopes are, in units of $10^{-19} \mathrm{~cm}^{2}$, 25.7 for ${ }^{57} \mathrm{Fe}, 14.0$ for ${ }^{119} \mathrm{Sn}$, and 1.14 for ${ }^{151} \mathrm{Eu}$. Finally, natural linewidths, $\Gamma$, of Equation 1, are $0.097 \mathrm{~mm} / \mathrm{s}$ for ${ }^{57} \mathrm{Fe}, 0.323 \mathrm{~mm} / \mathrm{s}$ for ${ }^{119} \mathrm{Sn}$, and $0.652 \mathrm{~mm} / \mathrm{s}$ for ${ }^{151} \mathrm{Eu}$.

The observed intensity in a Mössbauer transmission spectrum appears as a dip in count rate as the Mössbauer effect removes $\gamma$ rays from the transmitted beam. Since this dip in transmission increases with sample thickness, thicker samples provide better signal-to-noise ratios and shorter data acquisition times. For quantitative work, however, it is poor practice to work with samples that are the natural thickness, $t$, or thicker owing to an effect called "thickness distortion." In a typical constant- acceleration spectrometer, the incident beam will have uniform intensity at all velocities of the source, and the top layer of sample will absorb $\gamma$ rays in proportion to its cross-section (Equation 2). On the other hand, layers deeper within the sample will be exposed to a $\gamma$-ray intensity diminished at velocities where the top layers have absorbed strongly. The effect of this "thickness distortion" is to reduce the overall sample absorption at velocities where the Mössbauer effect is strong. Broadening of the Mössbauer peaks therefore occurs as the samples become thicker. This broadening can be modeled approximately as increasing the effective linewidth of Equation 1 from the natural $t$ to $\Gamma(1+0.135 \mathrm{t} /$ $t$ ), where $t$ is the sample thickness in units of $t$. However, it is important to note that in the tails of the Mössbauer peaks, where the absorption is weak, there is less thickness distortion. The peak shape in the presence of thickness distortion is therefore not a true Lorentzian function. Numerical corrections for the effects of thickness distortion are sometimes possible, but are rarely performed owing to difficulties in knowing the precise sample thickness and thickness uniformity. For quantitative work the standard practice is to use samples of thickness $t / 2$ or so.

We calculate an effective thickness, $t$, for the case of natural Fe metal, which is widely used as a calibration standard for Mössbauer spectrometers. If there are no impurities in the $\mathrm{Fe}$ metal, its Mössbauer spectrum has sharp lines as shown in Figure 2. The recoil-free-fraction of bcc $\mathrm{Fe}$ is 0.80 at $300 \mathrm{~K}$, and other quantities follow Equation 29

$$
\begin{gathered}
=\left(0.80 \times 0.022 \times \frac{7.86 \mathrm{~g}}{\mathrm{~cm}^{3}} \times \frac{1 \mathrm{~mol}}{55.85 \mathrm{~g}} \times\right. \\
\left.\frac{6.023 \times 10^{23} \text { atoms }}{\text { mole }} \times 25 \times 10^{-19} \mathrm{~cm}^{2} \frac{1}{4}\right)^{-1} \\
=11 \times 10^{-4} \mathrm{~cm}=11 \mu \mathrm{m} .
\end{gathered}
$$

The final factor of 1/4 in Equation 30 accounts for the fact that the absorption cross-section is split into six different peaks owing to the hyperfine magnetic field in bcc Fe. The strongest of these comprises $1 / 4$ of the total absorption. Figure 2 was acquired with a sample of natural bcc Fe 25 $\mu \mathrm{m}$ in thickness. The linewidths of the inner two peaks are $0.235 \mathrm{~mm} / \mathrm{s}$ whereas the outer two are $0.291 \mathrm{~mm} / \mathrm{s}$. Although the outer two peaks are broadened by thickness distortion, effects of impurity atoms in the $\mathrm{Fe}$ were also important. The widths of the inner two lines are probably a better measure of the spectrometer resolution.

\section{LITERATURE CITED}

Akai, H., Blügel, S., Zeller, R., and Dederichs, P. H., 1986. Isomer shifts and their relation to charge transfer in dilute Fe alloys. Phys. Rev. Lett. 56:2407-2410.

Bell, S. H., Weir, M. P., Dickson, D. P. E, Gibson, J. F., Sharp, G. A., and Peters, T. J. 1984. Mössbauer spectroscopic studies of human haemosiderin and ferritin. Biochim. Biophys. Acta. 787:227-236.

Black, P. J., and Moon, P. B. 1960. Resonant scattering of the 14-keV iron-57 $\gamma$-ray, and its interference with Rayleigh scattering. Nature (London) 188:481-484.

Bowen, L. H., De Grave, E., and Vandenberghe, R. E. 1993. Mössbauer effect studies of magnetic soils and sediments. In Mössbauer Spectroscopy Applied to Magnetism and Materials Science Vol. I. (G. J. Long and F. Grandjean, eds.). pp. 115-159. Plenum, New York.

Brand, R. A. and Le Car, G. 1988. Improving the validity of Mössbauer hyperfine parameter distributions: The maximum entropy formalism and its applications. $\mathrm{Nucl}$. Instr. Methods Phys. Res. B 34:272-284.

Campbell, S. J., Kaczmarek, W. A., and Wang, G. M. 1995. Mechanochemical transformation of haematite to magnetite. Nanostruct. Mater. 6:35-738.

Cortie, M. B. and Pollak, H. 1995. Embrittlement and aging at $475^{\circ} \mathrm{C}$ in an experimental ferritic stainless-steel containing 38 wt.\% chromium. Mater. Sci. Eng. A 199:153-163.

Cuenya, B. Roldan, Naitabdi, A., Croy, J., Sturhahn, W., Zhao, J.Y., Alp, E.E., Meyer, R., Sudfeld, D., Schuster, E., Keune, W. 2007. Atomic vibrations in iron nanoclusters: Nuclear resonant inelastic x-ray scattering and molecular dynamics simulations. Phys. Rev. B, 76:195422.

Cuenya, B. Roldan, Keune, W., Peters, R., Schuster, E., Sahoo, B., von Horsten, U., Sturhahn, W., Zhao, J., Toellner, T.S., Alp, E.E., Bader, S.D. 2008. High-energy phonon confinement in nanoscale metallic multilayers. Phys. Rev. B, 77:165410.

Degrave, E., Persoons, R. M., and Vandenberghe, R. E. 1993. Mössbauer study of the high temperature phase of co-substituted magnetite. Phys. Rev. B 47:5881-5893.

Dickson, D. P. E. 1987. Mössbauer Spectroscopic Studies of Magnetically Ordered Biological Materials. Hyperfine Interact. 33: 263-276.

Dodd, J.L., Halevy, I. and Fultz, B. 2007. Valence Fluctuations of ${ }^{57} \mathrm{Fe}$ in Disordered $\mathrm{Li}_{0.6} \mathrm{FePO}_{4}$. J. Phys. Chem C 111: 1563-1566. 
Faldum, T., Meisel, W., and Gütlich, P. 1994. Oxidic and metallic $\mathrm{Fe} / \mathrm{Ni}$ multilayers prepared from LangmuirBlodgett films. Hyperfine Interact. 92: 1263-1269.

Feldwisch, R., Sepiol, B., and Vogl, G. 1994. Elementary diffusion jump of iron atoms in intermetallic phases studied by Mössbauer spectroscopy 2. From order to disorder. Acta Metall. Mater. 42:3175-3181.

Fultz, B. 1982. A Mössbauer Spectrometry Study of Fe-Ni$\mathrm{X}$ Alloys. Ph.D. Thesis. University of California at Berkeley.

Fultz, B. 1993. Chemical systematics of iron-57 hyperfine magnetic field distributions in iron alloys. In Mössbauer Spectroscopy Applied to Magnetism and Materials Science Vol. I. (G. J. Long and F. Grandjean, eds.). pp. 1-31. Plenum Press, New York.

Fultz, B., Ahn, C. C, Alp, E. E., Sturhahn, W., and Toellner, T. S. 1997. Phonons in nanocrystalline ${ }^{57} \mathrm{Fe}$. Phys. Rev. Lett. 79: 937-940.

Fultz, B., Kuwano, H., and Ouyang, H. 1995. Average widths of grain boundaries in nanophase alloys synthesized by mechanical attrition. J. Appl. Phys. 77:3458-3466.

Gancedo, J. R., Gracia, M. and Marco, J. F. 1991. CEMS Methodology. Hyperfine Interact. 66:83-94.

Gerdau, E., Rüffer, R., Winkler, H., Tolksdorf, W., Klages, C. P., and Hannon, J. P. 1985. Nuclear Bragg diffraction of synchrotron radiation in yttrium iron garnet, Phys. Rev. Lett. 54: 835-838.

Greenwood, N. N. and Gibb, T. C. 1971. Mössbauer Spectroscopy. Chapman \& Hall, London.

Gütlich, P. 1975. Mössbauer spectroscopy in chemistry. In Mössbauer Spectroscopy. (U. Gonser, ed.). Chapter 2. Springer-Verlag, New York.

Hannon, J. P. and Trammell, G. T. 1969. Mössbauer diffraction. II. Dynamical Theory of Mössbauer Optics. Phys. Rev. 186:306-325.

Herr, U., Jing, J., Birringer, R., Gonser, U., and Gleiter, H. 1987. Investigation of nanocrystalline iron materials by Mössbauer spectroscopy. Appl. Phys. Lett. 50:472-474.

Hesse, J. and Rutbartsch, A. 1974. Model independent evaluation of overlapped Mössbauer spectra. J. Phys. E: Sci. Instrum, 7: 526-532.

Kruijer, S., Keune, W., Dobler, M., and Reuther, H. 1997. Depth analysis of phase formation in Si after high-dose $\mathrm{Fe}$ ion-implantation by depth-selective conversion electron Mössbauer spectroscopy. Appl Phys. Lett. 70:2696-2698.

Lagarec, K. and Rancourt, D. G. 1997. Extended Voigtbased analytic lineshape method for determining ndimensional correlated hyperfine parameter distributions in Mössbauer spectroscopy. Nucl. Inst. Methods Phys. Res. B 128:266-280.

Lamb, W. E. Jr. 1939. Capture of neutrons by atoms in a crystal. Phys. Rev. 55:190-197.

Le Caër, G. and Duboise, J. M. 1979. Evaluation of hyperfine parameter distributions from overlapped Mössbauer spectra of amorphous alloys. J. Phys. E: Sci. Instrum. 12:1083-1090.

Lin, J. Y. Y. and Fultz, B. 2003. Site-Specific Long-Range
Order in ${ }^{57} \mathrm{Fe}_{3} \mathrm{Al}$ Measured by Mössbauer

Diffractometry. Philos. Mag. 83:2621-2640.

Lin, J. Y. Y. and Fultz, B. 2004. Mössbauer Diffractometry Measurements of Site-Specific Long-Range Order in

${ }^{57} \mathrm{Fe}_{3} \mathrm{Al}$. Z. Kristallog. 219:172-178.

Mørup, S. 1990. Mössbauer effect in small particles. Hyperfine Interact. 60:959-974.

Mössbauer, R. L. 1958. Kernresonanzfluoreszenz von Gamma-strahlung in $\operatorname{Ir}^{191}$. Z. Phys. 151:124.

Ruebenbauer, K., Mullen, J. G., Nienhaus, G. U., and Shupp, G. 1994. Simple model of the diffusive scattering law in glass-forming liquids. Phys. Rev. B 49:15607-15614.

Schwartz, L. H. 1976. Ferrous alloy phase transformations. In Applications of Mössbauer Spectroscopy, Vol. 1. pp. 37-81. (R. L. Cohen, ed.). Academic Press, New York.

Seto, M., Yoda, Y., Kikuta, S., Zhang, X. W., and Ando, M. 1995. Observation of nuclear resonant scattering accompanied by phonon excitation using synchrotron radiation. Phys. Rev. Lett 74:3828-3831.

Sepiol, B., Meyer. A, Vogl, G., Ruffer, R., Chumakov, A. I., and Baron, A. Q. R. 1996. Time domain study of Fe57 diffusion using nuclear forward scattering of synchrotron radiation. Phys. Rev. Lett. 76:3220-3223.

Shvyd'ko, Yu. V., and Smirnov, G. V. 1989. Experimental study of time and frequency properties of collective nuclear excitations in a single crystal. J. Phys. Condens. Matter 1:10563-10584.

Simmons, G. W. and Leidheiser, Jr., H. 1976. Corrosion and interfacial reactions. In Applications of Mössbauer Spectroscopy, Vol 1. pp. 92-93. (R. L. Cohen, ed.). Academic Press, New York.

Smirnov, G. V. 1996. Nuclear resonant scattering of synchrotron radiation. Hyperfine Interact. 97/98:551588.

Squires, G. L. 1978. Introduction to the Theory of Thermal Neutron Scattering, p. 54. Dover Publications, New York.

Stahl, B. and Kankeleit, E. 1997. A high-luminosity UHV orange type magnetic spectrometer developed for depthselective Mössbauer spectroscopy. Nucl. Instr. Meth. Phys. Res. B 122: 149-161.

Stephens, T. A. and Fultz, B. 1997. Chemical environment selectivity in Mössbauer diffraction from ${ }^{57} \mathrm{Fe}_{3} \mathrm{Al}$. Phys. Rev. Lett. 78:366-369.

Stephens, T. A., Keune, W., and Fultz, B. 1994. Mössbauer effect diffraction from polycrystalline ${ }^{57} \mathrm{Fe}$. Hyperfine Interact. 92: 1095-1100.

Sturhahn, W., Toellner, T. S., Alp, E. E., Zhang, X., Ando, M., Yoda, Y., Kikuta, S., Seto, M., Kimball, C. W., and Dabrowski, B. 1995. Phonon density-of-states measured by inelastic nuclear resonant scattering. Phys. Rev. Lett. 74: 3832-3835.

Sturhahn, W. and Gerdau, E. 1994. Evaluation of TimeDifferential Measurements of Nuclear-Resonance Scattering of X-Rays. Phys. Rev. B 49: 9285-9294.

van Bürck, U., Smirnov, G. V., Mössbauer, R. L., Parak, F., and Semioschkina, N. A. 1978. Suppression of nuclear inelastic channels in nuclear resonance and electronic scattering of $\gamma$-quanta for different hyperfine transtions 
in perfect ${ }^{57} \mathrm{Fe}$ single crystals. J. Phys. C Solid State Phys. 11:2305-2321.

Vincze, I. and Aldred, A. T. 1974. Mössbauer measurements in iron-base alloys with nontransition elements. Phys. Rev. B 9:3845-3853.

Vincze, I. and Campbell, I. A. 1973. Mössbauer measurements in iron based alloys with transition metals. J. Phys. F Metal Phys. 3:647-663.

Vogl, G, and Sepiol, B. 1994. Elementary diffusion jump of iron atoms in intermetallic phases studied by Mössbauer spectroscopy 1. Fe-Al close to equiatomic stoichiometry. Acta Metall. Mater. 42:3175-3181.

Walker, L. R., Wertheim, G. K., and Jaccarino, V. 1961. Interpretation of the $\mathrm{Fe}^{57}$ isomer shift. Phys. Rev. Lett. 6:98-101.

Watson, R. E. and Freeman, A. J. 1967. Hartree-Fock theory of electric and magnetic hyperfine interactions in atoms and magnetic compounds. In Hyperfine Interactions. (A. J. Freeman and R. B. Frankel, eds.) Chapter 2. Academic Press, New York.

Williamson, D. L. 1993. Microstructure and tribology of carbon, nitrogen, and oxygen implanted ferrous materials. Nucl. Instr. Methods Phys. Res. B 76:262267.

\section{KEY REFERENCES}

Bancroft, G. M. 1973. Mössbauer Spectroscopy: An Introduction for Inorganic Chemists and Geochemists. John Wiley \& Sons, New York.

Belozerski, G. N. 1993. Mössbauer Studies of Surface Layers. Elsevier/North Holland, Amsterdam.

Cohen, R. L. (ed.). 1980. Applications of Mössbauer Spectroscopy, Vols. 1,2. Academic Press, New York.

These 1980 volumes by Cohen contain review articles on the applications of Mössbauer spectrometry to a wide range of materials and phenomena, with some exposition of the principles involved.

Cranshaw, T. E., Dale, B. W., Longworth, G. O., and Johnson, C. E. 1985. Mössbauer Spectroscopy and its Applications. Cambridge University Press, Cambridge.

Dickson, D. P. E. and Berry, F. J. (eds.). 1986. Mössbauer Spectroscopy. Cambridge University Press, Cambridge.

The book edited by Dickson and Berry contains detailed review articles on inorganic materials, physical chemistry, magnetic materials, and dynamic phenomena.

Frauenfelder, H. 1962. The Mössbauer Effect: A Review with a Collection of Reprints. W. A. Benjamin, New York.

This book by Frauenfelder was written in the early days of Mössbauer spectrometry, but contains a fine exposition of principles. More importantly, it contains reprints of the papers that first reported the phenomena that are the basis for much of Mössbauer spectrometry. It includes an English translation of one of Mössbauer's first papers.

Gibb, T. C. 1976. Principles of Mössbauer Spectroscopy. Chapman and Hall, London.
Gonser, U. (ed.). 1975. Mössbauer Spectroscopy. SpringerVerlag, New York.

Gonser, U. (ed.). 1986. Microscopic Methods in Metals, Topics in Current Physics, 40, Springer-Verlag, Berlin.

Gruverman, I. J. (ed.). 1976. Mössbauer Effect Methodology, Vols. 1-10. Plenum Press, New York.

Gütlich, P., Link, R., and Trautwein, A. (eds.). 1978. Mössbauer Spectroscopy and Transition Metal Chemistry. Springer- Verlag, Berlin.

Long, G. J. and Grandjean, F. (eds.). 1984. Mössbauer Spectroscopy Applied to Inorganic Chemistry, Vols. 13 Plenum Press, New York.

Long, G. J. and Grandjean, F. (eds.). 1996. Mössbauer Spectroscopy Applied to Magnetism and Materials Science, Vols. 1 and 2. Plenum Press, New York.

These 1996 volumes by Long and Grandjean contain review articles on different classes of materials, and on different techniques used in Mössbauer spectrometry.

Long, G. J. and Stevens, J. G. (eds.). 1986. Industrial Applications of the Mössbauer Effect. Plenum, New York.

May, L. (ed.). 1971 An Introduction to Mössbauer Spectroscopy Plenum Press, New York.

The short book by May has an intuitive explanation of the Mössbauer effect that is popular as a first explanation of the phenomenon.

Mitra, S. (ed.). 1992. Applied Mössbauer Spectroscopy: Theory and Practice for Geochemists and Archaeologists. Pergamon Press, Elmsford, New York.

Thosar, B. V. and Iyengar, P. K. (eds.). 1983 Advances in Mössbauer Spectroscopy, Studies in Physical and Theoretical Chemistry 25. Elsevier/North Holland, Amsterdam.

Wertheim, G. 1964. Mössbauer Effect: Principles and Applications. Academic Press, New York.

\section{INTERNET RESOURCES}

http://www.kfki.hu/ mixhp/

The Mössbauer Information eXchange, MIX, is a project of the KFKI Research Institute for Particle and Nuclear Physics, Budapest, Hungary. It is primarily for scientists, students, and manufacturers involved in Mössbauer spectroscopy and other nuclear solid-state methods.

http://www.medc.dicp.ac.cn

http://www.mossbauer.org/

The Mössbauer Effect Data Center (Dalian Institute of Chemical Physics, China) maintains a library of most publications involving the Mössbauer effect, including hard-to-access publications. Computerized databases and database search services are available to find papers on specific materials.
BRENT FULTZ

California Institute of Technology

Pasadena, California 